\title{
Convergence analysis of new modified iterative approximating processes for two finite families of total asymptotically nonexpansive nonself mappings in hyperbolic spaces
}

\author{
Ting-jian Xiong, Heng-you Lan* \\ Department of Mathematics, Sichuan University of Science \& Engineering, 643000 Zigong, Sichuan, P. R. China. \\ Communicated by Y. J. Cho
}

\begin{abstract}
In this paper, we introduce and study a class of new modified iterative approximation processes for two finite families of total asymptotically nonexpansive nonself mappings in hyperbolic spaces. By using generalization of Schu's lemma and Tan-Xu's inequality, some important related properties of this modified iterative approximation are proposed and analyzed. Further, based on the related properties, we prove $\Delta$-convergence and strong convergence of the modified iterative approximating process in hyperbolic spaces. Because a total asymptotically nonexpansive nonself mapping in hyperbolic spaces includes asymptotically nonexpansive mapping, (generalized) nonexpansive mapping of all normed linear spaces, Hadamard manifolds and CAT(0) spaces as special cases, the results presented in this paper improve and generalize the corresponding results in the literature. (C) 2017 All rights reserved.
\end{abstract}

Keywords: Convergence analysis, new modified iterative approximating process, $\Delta$-convergence and strong convergence, total asymptotically nonexpansive nonself mapping, hyperbolic space.

2010 MSC: 47H09, 47H10, 54E70.

\section{Introduction and preliminaries}

In this paper, we consider the following new modified iterative approximating process in a hyperbolic space $X$ :

$$
\begin{aligned}
y_{n} & =P W\left(T_{r}\left(P T_{r}\right)^{n-1} x_{n}, W\left(x_{n}, S_{r}\left(P S_{r}\right)^{n-1} x_{n}, \theta_{r n}\right), \alpha_{r n}\right), \\
y_{n+1} & =P W\left(T_{r-1}\left(P T_{r-1}\right)^{n-1} y_{n}, W\left(y_{n}, S_{r-1}\left(P S_{r-1}\right)^{n-1} y_{n}, \theta_{(r-1) n}\right), \alpha_{(r-1) n}\right), \\
& \vdots \\
y_{n+r-3}= & P W\left(T_{3}\left(P T_{3}\right)^{n-1} y_{n+r-4}, W\left(y_{n+r-4}, S_{3}\left(P S_{3}\right)^{n-1} y_{n+r-4}, \theta_{3 n}\right), \alpha_{3 n}\right), \\
y_{n+r-2}= & P W\left(T_{2}\left(P_{2}\right)^{n-1} y_{n+r-3}, W\left(y_{n+r-3}, S_{2}\left(P S_{2}\right)^{n-1} y_{n+r-3}, \theta_{2 n}\right), \alpha_{2 n}\right), \\
x_{n+1}= & P W\left(T_{1}\left(P T_{1}\right)^{n-1} y_{n+r-2}, W\left(y_{n+r-2}, S_{1}\left(P S_{1}\right)^{n-1} y_{n+r-2}, \theta_{1 n}\right), \alpha_{1 n}\right),
\end{aligned}
$$

\footnotetext{
*Corresponding author

Email address: hengyoulan@163.com (Heng-you Lan)
} 
where $\theta_{i n}=\frac{\beta_{i n}}{1-\alpha_{i n}}$ for $\alpha_{i n}, \beta_{i n} \in(0,1)$ and all $i \in \mathfrak{R}=\{1,2, \cdots, r\}$ and $n \geqslant 1,\left\{T_{i}\right\}_{i \in \mathfrak{R}}$ and $\left\{S_{i}\right\}_{i \in \mathfrak{R}}$ are two finite families of nonlinear mappings on $\mathrm{K}$ onto $\mathrm{X}$ and $\mathrm{P}: \mathrm{X} \rightarrow \mathrm{K} \subset \mathrm{X}$ is a nonexpansive retraction.

Remark 1.1. For appropriate and suitable choices of the nonlinear mappings $T_{i}, S_{i}$, real numbers $\alpha_{i n}, \beta_{i n}$ $(i \in \mathfrak{R})$, positive integer $r$, mapping $P$ and the space $X$, the sequence $\left\{x_{n}\right\}$ generated by (1.1) includes a number of known and investigated iterative schemas. See, for example, $[1,2,4,5,8,12,14,16,19-$ $21,24,27-31,33$ ] and the references therein, and the following examples:

Example 1.2. If $P=I$ (the identity mapping) and $T_{i}$ and $S_{i}$ are self-mappings from $K$ to $K$ for all $i=$ $1,2, \cdots, r$, then $(1.1)$ reduces to

$$
\begin{aligned}
y_{n} & =W\left(T_{r}^{n} x_{n}, W\left(x_{n}, S_{r}^{n} x_{n}, \theta_{r n}\right), \alpha_{r n}\right), \\
y_{n+1} & =W\left(T_{r-1}^{n} y_{n}, W\left(y_{n}, S_{r-1}^{n} y_{n}, \theta_{(r-1) n}\right), \alpha_{(r-1) n}\right), \\
& \vdots \\
y_{n+r-3} & =W\left(T_{3}^{n} y_{n+r-4}, W\left(y_{n+r-4}, S_{3}^{n} y_{n+r-4}, \theta_{3 n}\right), \alpha_{3 n}\right), \\
y_{n+r-2} & =W\left(T_{2}^{n} y_{n+r-3}, W\left(y_{n+r-3}, S_{2}^{n} y_{n+r-3}, \theta_{2 n}\right), \alpha_{2 n}\right), \\
x_{n+1} & =W\left(T_{1}^{n} y_{n+r-2}, W\left(y_{n+r-2}, S_{1}^{n} y_{n+r-2}, \theta_{1 n}\right), \alpha_{1 n}\right),
\end{aligned}
$$

which was considered by Fukhar-ud-din and Khan [4] when $\left\{T_{i}\right\}_{i \in \mathfrak{R}}$ and $\left\{S_{i}\right\}_{i \in \mathfrak{R}}$ are two families of asymptotically quasi-nonexpansive self-mappings on $\mathrm{K}$.

Example 1.3. When $r=3$ and $\alpha_{i n}=0,(1.2)$ is equivalent to the iterative process introduced and studied by Noor [16], which was dealt with variational inequalities in Hilbert spaces. Moreover, Sahin and Basarir [20] considered a unified treatment regarding iterative process for total asymptotically nonexpansive mapping in hyperbolic spaces. For more detail, refer to $[19,24]$ and the references therein.

Example 1.4. Let $r=2, \alpha_{1 n}=\beta_{1 n}=0$, and $\alpha_{2 n}=0$, then (1.2) becomes to the iteration discussed by Thakur et al. [27] in $\operatorname{CAT}(0)$ space as follows:

$$
x_{n+1}=S_{1}^{n} y_{n}, \quad y_{n}=W\left(x_{n}, S_{2}^{n} x_{n}, \beta_{2 n}\right) .
$$

The iteration (1.3) is called a modified hybrid Picard-Mann iterative process, and includes Picard-Mann iteration introduced firstly by Khan [5] in 2013.

Example 1.5. If $r=1$ and $\alpha_{1 n}=0$ (or $r=2, \alpha_{1 n}=0, \beta_{1 n}=1$ and $\alpha_{2 n}=0$ ), then (1.2) is equivalent to

$$
x_{n+1}=W\left(x_{n}, S_{1}^{n} x_{n}, \beta_{1 n}\right) \quad\left(\text { or } x_{n+1}=W\left(x_{n}, S_{2}^{n} x_{n}, \beta_{2 n}\right)\right),
$$

which is well-known modified Mann iteration, and was introduced and considered by Schu [21] in Banach spaces.

In order to explore an iterative approximation for the process generated by (1.1), now we recall that a hyperbolic space introduced by Kohlenbach [9], is a metric space $(X, d)$ together with a mapping $W$ : $X^{2} \times[0,1] \rightarrow X$ satisfying the following conditions: for all $u, x, y, z, w \in X$ and $\alpha, \beta \in[0,1]$,

(i) $d(u, W(x, y, \alpha)) \leqslant \alpha d(u, x)+(1-\alpha) d(u, y)$;

(ii) $d(W(x, y, \alpha), W(x, y, \beta))=|\alpha-\beta| d(x, y)$;

(iii) $W(x, y, \alpha)=W(y, x,(1-\alpha))$;

(iv) $d(W(x, z, \alpha), W(y, w, \alpha)) \leqslant \alpha d(x, y)+(1-\alpha) d(z, w)$.

We note that a metric space which satisfies only condition (i), is consistent with a convex metric space, which was introduced by Takahashi [25]. Further, the concept of hyperbolic spaces in [9] is more restrictive than that introduced by Kuhfittig [11], because conditions (i)-(iii) together are equivalent to $(X, d, W)$ being a space of hyperbolic type in [11]. But the hyperbolic spaces in [9] are slightly more general than that 
defined by Reich and Shafrir [18]. However, the class of hyperbolic spaces in the sense of Kohlenbach [9] contains all normed linear spaces and convex subsets thereof but also Hadamard manifolds and CAT $(0)$ spaces. For more on hyperbolic spaces and a comparison between different notions of hyperbolic space, and some examples of hyperbolic spaces one can refer to $[3,9,31]$ and the references therein.

Definition 1.6. Let $X$ be a hyperbolic space and $K \subset X$ be a nonempty subset, and let $\eta:(0,+\infty) \times(0,2] \rightarrow$ $(0,1]$ be a nonlinear mapping. Then

(i) $K$ is convex if $W(x, y, \alpha) \in K$ for all $x, y \in K$ and $\alpha \in[0,1]$;

(ii) $X$ is uniformly convex if for any $r>0$ and $\epsilon \in(0,2]$, and all $u, x, y \in X$, there exists $\delta \in(0,1]$ such that

$$
d\left(W\left(x, y, \frac{1}{2}\right), u\right) \leqslant(1-\delta) r
$$

with $\max \{d(x, u), d(y, u)\} \leqslant r$ and $d(x, y) \geqslant r \in($ see $[3,23])$;

(iii) A modulus of uniform convexity of $X$ is denoted by $\delta=\eta(r, \epsilon)$ for given $r>0$ and $\epsilon \in(0,2]$;

(iv) $\eta$ is monotone if it decreases with respect to $r$, i.e.,

$$
\eta\left(r_{2}, \epsilon\right) \leqslant \eta\left(r_{1}, \epsilon\right), \quad \forall \epsilon>0, r_{2} \geqslant r_{1}>0 .
$$

Remark 1.7. A CAT(0) space is a uniformly convex hyperbolic space when $\eta(r, \varepsilon)=\frac{\varepsilon^{2}}{8}$ (see [13]). Thus, the class of uniformly convex hyperbolic spaces includes both uniformly convex normed spaces and CAT $(0)$ spaces as special cases.

In the sequel, let $(X, d)$ be a metric space and $K \subset X$ be nonempty. We shall denote the fixed point set of a nonself-mapping $T: K \rightarrow X$ by $F(T)=\{x \in K: T x=x\}$. Recall that $K$ is said to be a retract of $X$ if there exists a continuous mapping $P: X \rightarrow K$ such that $P x=x$ for all $x \in K$. A mapping $P: X \rightarrow K$ is said to be a retraction if $P^{2}=P$. If $P$ is a retraction, then $P y=y$ for $y$ in the range of $P$.

Definition 1.8. Let $P: X \rightarrow K$ be a nonexpansive retraction. A mapping $T: K \rightarrow X$ is said to be

(i) nonexpansive if

$$
\mathrm{d}(T x, T y) \leqslant d(x, y), \quad \forall x, y \in K ;
$$

(ii) asymptotically nonexpansive if there exists a sequence $\left\{k_{n}\right\} \subset[0,+\infty)$ with $\lim _{n \rightarrow \infty} k_{n}=0$ such that

$$
d\left(T^{n} x, T^{n} y\right) \leqslant\left(1+k_{n}\right) d(x, y), \quad \forall x, y \in K, n \geqslant 1 ;
$$

(iii) asymptotically quasi-nonexpansive if there exists $\left\{k_{n}\right\}$ as in (1.4) such that

$$
d\left(T^{n} x, p\right) \leqslant\left(1+k_{n}\right) d(x, p), \quad \forall x \in K, p \in F(T) \neq \emptyset, n \geqslant 1 ;
$$

(iv) $\left(\left\{\mu_{n}\right\},\left\{\xi_{n}\right\}, \rho\right)$-total asymptotically nonexpansive if there exist nonnegative sequences $\left\{\mu_{n}\right\},\left\{\xi_{n}\right\}$ and a strictly increasing continuous function $\rho:[0,+\infty) \rightarrow[0,+\infty)$ with $\mu_{n} \rightarrow 0, \xi_{n} \rightarrow 0$ and $\rho(0)=0$ such that

$$
d\left(T^{n} x, T^{n} y\right) \leqslant d(x, y)+\mu_{n} \rho(d(x, y))+\xi_{n}, \quad \forall x, y \in K, n \geqslant 1 ;
$$

(v) $\left(\left\{\mu_{n}\right\},\left\{\xi_{n}\right\}, \rho\right)$-total asymptotically nonexpansive nonself-mapping if there exist $\left\{\mu_{n}\right\},\left\{\xi_{n}\right\}$ and $\rho$ as in (1.5) such that

$$
d\left(T(P T)^{n-1} x, T(P T)^{n-1} y\right) \leqslant d(x, y)+\mu_{n} \rho(d(x, y))+\xi_{n}, \quad \forall x, y \in K, n \geqslant 1 ;
$$

(vi) $\left(\left\{\mu_{n}\right\},\left\{\xi_{n}\right\}, \rho\right)$-total asymptotically quasi-nonexpansive nonself-mapping if there exist $\left\{\mu_{n}\right\},\left\{\xi_{n}\right\}$ and $\rho$ as in (1.5) such that

$$
d\left(T(P T)^{n-1} x, p\right) \leqslant d(x, p)+\mu_{n} \rho(d(x, p))+\xi_{n}, \quad \forall x \in K, p \in F(T) \neq \emptyset, n \geqslant 1 ;
$$


(vii) uniformly L-Lipschitzian if there exists a constant $L>0$ such that

$$
d\left(T(P T)^{n-1} x, T(P T)^{n-1} y\right) \leqslant \operatorname{Ld}(x, y), \quad \forall x, y \in K, n \geqslant 1 .
$$

Remark 1.9. By Definition 1.8, one can know that a nonexpansive mapping is an asymptotically nonexpansive mapping with $k_{n} \equiv 0$ for $n \geqslant 1$, and each asymptotically nonexpansive mapping is a $\left(\left\{\mu_{n}\right\},\left\{\xi_{n}\right\}, \rho\right)$ total asymptotically nonexpansive mapping with $\xi_{n}=0$, and $\rho(t)=t \geqslant 0$. However, in general, the converse is not true. Indeed, Wang et al. [30] introduced the class of total asymptotically nonexpansive nonself-mappings, and generalized and extended several classes of asymptotically nonexpansive mappings. For more detail, see, for example, $[29,32]$ and the references therein.

As all we know that uniformly convex Banach spaces and even CAT(0) spaces enjoy the property that "bounded sequences have unique asymptotic centers with respect to closed convex subsets". The following lemma is due to Leustean [13] and ensures that this property also holds in a complete uniformly convex hyperbolic space.

Lemma 1.10 ([13]). Let $(\mathrm{X}, \mathrm{d}, \mathrm{W})$ be a complete uniformly convex hyperbolic space with monotone modulus of uniform convexity. Then every bounded sequence $\left\{x_{n}\right\} \subset X$ has a unique asymptotic center with respect to any nonempty closed convex subset $\mathrm{K} \subset \mathrm{X}$.

Lemma 1.11 ([7]). Let $(\mathrm{X}, \mathrm{d}, \mathrm{W})$ be a uniformly convex hyperbolic space with monotone modulus of uniform convexity $\eta$. Let $x \in X$ and sequence $\left\{\alpha_{n}\right\} \subset[a, b]$ for some $a, b \in(0,1)$. If $\left\{x_{n}\right\}$ and $\left\{y_{n}\right\}$ are sequences in $X$ such that for some $\mathrm{c} \geqslant 0$,

$$
\limsup _{n \rightarrow \infty} d\left(x_{n}, x\right) \leqslant c, \quad \limsup _{n \rightarrow \infty} d\left(y_{n}, x\right) \leqslant c, \quad \lim _{n \rightarrow \infty} d\left(W\left(x_{n}, y_{n}, \alpha_{n}\right), x\right)=c,
$$

then $\lim _{n \rightarrow \infty} d\left(x_{n}, y_{n}\right)=0$.

Lemma 1.12 ([26]). Let $\left\{a_{n}\right\},\left\{b_{n}\right\}$, and $\left\{\omega_{n}\right\}$ be nonnegative real sequences satisfying

$$
a_{n+1} \leqslant\left(1+\omega_{n}\right) a_{n}+b_{n}, \quad \forall n \geqslant 1 .
$$

If $\sum_{n=1}^{\infty} \omega_{n}<\infty$ and $\sum_{n=1}^{\infty} b_{n}<\infty$, then $\lim _{n \rightarrow \infty} a_{n}$ exists. Further, if there exists a subsequence $\left\{a_{n_{i}}\right\} \subset\left\{a_{n}\right\}$ such that $\mathrm{a}_{\mathrm{n}_{\mathrm{i}}} \rightarrow 0$, then $\lim _{\mathrm{n} \rightarrow \infty} \mathrm{a}_{\mathrm{n}}=0$.

Lemma 1.13 ([29]). Let $(X, d, W)$ be a complete uniformly convex hyperbolic space with monotone modulus of uniform convexity $\eta$, and $\mathrm{K}$ be a nonempty closed and convex subset of $\mathrm{X}$. Suppose that $\mathrm{T}: \mathrm{K} \rightarrow \mathrm{X}$ is a uniformly L-Lipschitzian and $\left(\left\{\mu_{n}\right\},\left\{\xi_{n}\right\}, \rho\right)$-total asymptotically nonexpansive nonself-mapping, and $\mathrm{P}$ is a nonexpansive retraction of $X$ onto $\mathrm{K}$. If $\left\{x_{n}\right\} \subset \mathrm{K}$ is a bounded approximate fixed point sequence, i.e., $\lim _{n \rightarrow \infty}\left(x_{n}, T x_{n}\right)=0$ and $\left\{x_{n}\right\} \rightarrow p$, then $T(p)=p$.

Inspired and motivated by the above recent works, in this paper, we shall study some important related properties of the new modified iterative approximating process (1.1) for two finite families of total asymptotically nonexpansive nonself-mappings as well as two finite families of total asymptotically quasinonexpansive nonself-mappings in hyperbolic spaces. Further, $\Delta$-convergence and strong convergence of this iterative process are proved.

\section{2. $\Delta$-convergence}

Throughout this paper, we assume that $\mathfrak{R}=\{1,2, \cdots, r\},\left\{T_{i}\right\}_{i \in \mathfrak{R}}$ and $\left\{S_{i}\right\}_{i \in \mathfrak{R}}$ are two finite families of total asymptotically (quasi-) nonexpansive nonself-mappings on $\mathrm{K}$ onto $\mathrm{X}, \mathrm{P}: \mathrm{X} \rightarrow \mathrm{K}$ is a nonexpansive retraction, and for each $i \in \mathfrak{R}$ and all $n \geqslant 1, \theta_{i n}=\frac{\beta_{i n}}{1-\alpha_{i n}},\left\{\alpha_{i n}\right\}$ and $\left\{\beta_{i n}\right\}$ are two double real sequences in $[a, b]$, i.e,

$$
0<a \leqslant \alpha_{i n}, \quad \beta_{i n} \leqslant b<1 .
$$


On behalf of defining the concept of $\Delta$-convergence in the general setup of hyperbolic spaces, we first give some basic concepts.

In 1976, Lim [15] proposed the notion of asymptotic center and original concept of $\Delta$-convergence in a general setting of a metric space. Recently, Kirk and Panyanak [10] introduced an analogous version of convergence in geodesic spaces (i.e., $\Delta$-convergence), and pointed out " $\Delta$-convergence coincides with the usual weak convergence in Banach spaces and both concepts share many useful properties".

Let $\left\{x_{n}\right\}$ be a bounded sequence in a hyperbolic space $X$. For $x \in X$, we define a continuous functional $r\left(\cdot,\left\{x_{n}\right\}\right): X \rightarrow[0,+\infty)$ by

$$
r\left(x,\left\{x_{n}\right\}\right)=\limsup _{n \rightarrow \infty} d\left(x, x_{n}\right) .
$$

The asymptotic radius $r\left(\left\{x_{n}\right\}\right)$ of $\left\{x_{n}\right\}$ is defined by $r\left(\left\{x_{n}\right\}\right)=\inf \left\{r\left(x,\left\{x_{n}\right\}\right): x \in X\right\}$. The asymptotic center of a bounded sequence $\left\{x_{n}\right\}$ with respect to $K \subset X$ is defined as follows:

$$
A_{K}\left(\left\{x_{n}\right\}\right)=\left\{x \in X: r\left(x,\left\{x_{n}\right\}\right) \leqslant r\left(y,\left\{x_{n}\right\}\right), \forall y \in K\right\},
$$

which is the set of minimizers for $r\left(\cdot,\left\{x_{n}\right\}\right)$. If the asymptotic center is taken with respect to $X$, then it is simply denoted by $A\left(\left\{x_{n}\right\}\right)$.

Definition 2.1. A sequence $\left\{x_{n}\right\} \subset X$ is called $\Delta$-converge to $x \in X$ if $x$ is the unique asymptotic center of $\left\{x_{n_{i}}\right\}$ for every subsequence $\left\{x_{n_{i}}\right\}$ of $\left\{x_{n}\right\}$.

By this time, we denote $\Delta$ - $\lim _{n \rightarrow \infty} x_{n}=x$ and call $x$ the $\Delta$-limit of $\left\{x_{n}\right\}$. In what follows, we indicate the notation $\left\{x_{n}\right\} \rightarrow p$ if and only if $\Phi(p)=\inf _{x \in K} \Phi(x)$, where $\Phi(x)=\limsup _{n \rightarrow \infty} d\left(x_{n}, x\right)$. We note that $\left\{x_{n}\right\} \rightarrow p$ is equivalent to $A_{K}\left(\left\{x_{n}\right\}\right)=\{p\}$, and $\Delta-\lim _{n \rightarrow \infty} x_{n}=p$ implies that $\left\{x_{n}\right\} \rightarrow p$ (see [29, 32]).

For discussing the new modified iterative approximating process (1.1), we present the following important related lemmas.

Lemma 2.2. Let $\mathrm{K}$ be a nonempty closed and convex subset of a hyperbolic space $X$. For $i \in \mathfrak{R}$, let $\mathrm{T}_{i}$ : $\mathrm{K} \rightarrow \mathrm{X}$ be a $\left(\left\{\mu_{\mathrm{n}}^{i}\right\},\left\{\xi_{n}^{i}\right\}, \rho^{i}\right)$-total asymptotically quasi-nonexpansive nonself-mapping with $\lim _{n \rightarrow \infty} \mu_{\mathrm{n}}^{i}=0$ and $\lim _{n \rightarrow \infty} \xi_{n}^{i}=0$, and a strictly increasing continuous function $\rho^{i}:[0,+\infty) \rightarrow[0,+\infty)$ satisfying $\rho^{i}(0)=0$, and let $S_{i}: K \rightarrow X$ be a $\left(\left\{\hat{\mu}_{n}^{i}\right\},\left\{\hat{\xi}_{n}^{i}\right\}, \hat{\rho}^{i}\right)$-total asymptotically quasi-nonexpansive nonself-mapping with $\lim _{n \rightarrow \infty} \hat{\mu}_{n}^{i}=0$ and $\lim _{n \rightarrow \infty} \hat{\xi}_{n}^{i}=0$, and a strictly increasing continuous function $\hat{\rho}^{i}:[0,+\infty) \rightarrow[0,+\infty)$ satisfying $\hat{\rho}^{i}(0)=0$. Assume that $\mathrm{F}=\bigcap_{i=1}^{r}\left(\mathrm{~F}\left(\mathrm{~T}_{i}\right) \cap \mathrm{F}\left(\mathrm{S}_{i}\right)\right) \neq \emptyset$, and for each $i \in \mathfrak{R}$, the following conditions hold:

(i) $\sum_{n=1}^{\infty} \mu_{n}^{i}<+\infty, \sum_{n=1}^{\infty} \hat{\mu}_{n}^{i}<+\infty, \sum_{n=1}^{\infty} \xi_{n}^{i}<+\infty, \sum_{n=1}^{\infty} \hat{\xi}_{n}^{i}<+\infty$.

(ii) There exist constants $M_{i}>0$ and $\hat{M}_{i}>0$ such that

$$
\rho^{i}(r) \leqslant M_{i} r, \quad \hat{\rho}^{i}(r) \leqslant \hat{M}_{i} r, \quad \forall r>0 .
$$

Then, for the sequence $\left\{x_{n}\right\}$ defined by (1.1), $\lim _{n \rightarrow \infty} d\left(x_{n}, p\right)$ exists for all $p \in F$.

Proof. Let $\mu_{n}=\max _{i \in \mathfrak{R}}\left\{\mu_{n}^{i}, \hat{\mu}_{n}^{i}\right\}$, and $\xi_{n}=\max _{i \in \mathfrak{R}}\left\{\xi_{n}^{i}, \hat{\xi}_{\mathfrak{n}}^{i}\right\}, \rho=\max _{i \in \mathfrak{R}}\left\{\rho^{i}, \hat{\rho}^{i}\right\}, M^{*}=\max _{i \in \mathfrak{R}}\left\{M_{i}, \hat{M}_{i}\right\}$. By conditions (i) and (ii), we have $\sum_{n=1}^{\infty} \mu_{n}<+\infty, \sum_{n=1}^{\infty} \xi_{n}<+\infty, \rho(r) \leqslant M^{*} r$ for any $r>0$. For every $p \in F$ and any $n \geqslant 1$, it follows from (1.1) that

$$
\begin{aligned}
d\left(y_{n}, p\right)= & d\left(P W\left(T_{r}\left(P T_{r}\right)^{n-1} x_{n}, W\left(x_{n}, S_{r}\left(P S_{r}\right)^{n-1} x_{n}, \theta_{r n}\right), \alpha_{r n}\right), P p\right) \\
\leqslant & d\left(W\left(T_{r}\left(P T_{r}\right)^{n-1} x_{n}, W\left(x_{n}, S_{r}\left(P S_{r}\right)^{n-1} x_{n}, \theta_{r n}\right), \alpha_{r n}\right), p\right) \\
\leqslant & \alpha_{r n} d\left(T_{r}\left(P T_{r}\right)^{n-1} x_{n}, p\right)+\left(1-\alpha_{r n}\right) d\left(W\left(x_{n}, S_{r}\left(P S_{r}\right)^{n-1} x_{n}, \theta_{r n}\right), p\right) \\
\leqslant & \alpha_{r n} d\left(T_{r}\left(P T_{r}\right)^{n-1} x_{n}, p\right)+\beta_{r n} d\left(x_{n}, p\right)+\left(1-\alpha_{r n}-\beta_{r n}\right) d\left(S_{r}\left(P S_{r}\right)^{n-1} x_{n}, p\right) \\
\leqslant & \alpha_{r n}\left[d\left(x_{n}, p\right)+\mu_{n}^{r} \rho^{r}\left(d\left(x_{n}, p\right)\right)+\xi_{n}^{r}\right]+\beta_{r n} d\left(x_{n}, p\right) \\
& +\left(1-\alpha_{r n}-\beta_{r n}\right)\left[d\left(x_{n}, p\right)+\hat{\mu}_{n}^{r} \hat{\rho}^{r}\left(d\left(x_{n}, p\right)\right)+\hat{\xi}_{n}^{r}\right] \\
\leqslant & \alpha_{r n}\left[d\left(x_{n}, p\right)+\mu_{n} \rho\left(d\left(x_{n}, p\right)\right)+\xi_{n}\right]+\beta_{r n} d\left(x_{n}, p\right) \\
& +\left(1-\alpha_{r n}-\beta_{r n}\right)\left[d\left(x_{n}, p\right)+\mu_{n} \rho\left(d\left(x_{n}, p\right)\right)+\xi_{n}\right] \\
\leqslant & {\left[1+\left(1-\beta_{r n}\right) \mu_{n} M^{*}\right] d\left(x_{n}, p\right)+\left(1-\beta_{r n}\right) \xi_{n} } \\
\leqslant & \left(1+\mu_{n} M^{*}\right) d\left(x_{n}, p\right)+\xi_{n}
\end{aligned}
$$


and

$$
\begin{aligned}
d\left(y_{n+1}, p\right)= & d\left(P W\left(T_{r-1}\left(P T_{r-1}\right)^{n-1} y_{n}, W\left(y_{n}, S_{r-1}\left(P S_{r-1}\right)^{n-1} y_{n}, \theta_{(r-1) n}\right), \alpha_{(r-1) n}\right), P p\right) \\
\leqslant & d\left(W\left(T_{r-1}\left(P T_{r-1}\right)^{n-1} y_{n}, W\left(y_{n}, S_{r-1}\left(P S_{r-1}\right)^{n-1} y_{n}, \theta_{(r-1) n}\right), \alpha_{(r-1) n}\right), p\right) \\
\leqslant & \alpha_{(r-1) n} d\left(T_{r-1}\left(P T_{r-1}\right)^{n-1} y_{n}, p\right)+\beta_{(r-1) n} d\left(y_{n}, p\right) \\
& +\left(1-\alpha_{(r-1) n}-\beta_{(r-1) n}\right) d\left(S_{r-1}\left(P S_{r-1}\right)^{n-1} y_{n}, p\right) \\
\leqslant & \alpha_{(r-1) n}\left[d\left(y_{n}, p\right)+\mu_{n} \rho\left(d\left(y_{n}, p\right)\right)+\xi_{n}\right]+\beta_{(r-1) n} d\left(y_{n}, p\right) \\
& +\left(1-\alpha_{(r-1) n}-\beta_{(r-1) n}\right)\left[d\left(y_{n}, p\right)+\mu_{n} \rho\left(d\left(y_{n}, p\right)\right)+\xi_{n}\right] \\
\leqslant & {\left[1+\left(1-\beta_{(r-1) n}\right) \mu_{n} M^{*}\right] d\left(y_{n}, p\right)+\left(1-\beta_{(r-1) n}\right) \xi_{n} } \\
\leqslant & \left(1+\mu_{n} M^{*}\right) d\left(y_{n}, p\right)+\xi_{n} .
\end{aligned}
$$

Similarly, we have

$$
d\left(y_{n+r-2}, p\right) \leqslant\left(1+\mu_{n} M^{*}\right) d\left(y_{n+r-3}, p\right)+\xi_{n}, \quad d\left(x_{n+1}, p\right) \leqslant\left(1+\mu_{n} M^{*}\right) d\left(y_{n+r-2}, p\right)+\xi_{n} .
$$

Thus,

$$
\begin{aligned}
d\left(x_{n+1}, p\right) \leqslant & \left(1+\mu_{n} M^{*}\right)^{r} d\left(x_{n}, p\right)+\sum_{j=0}^{r-1}\left(1+\mu_{n} M^{*}\right)^{j} \xi_{n} \\
\leqslant & d\left(x_{n}, p\right)\left[1+\left(\begin{array}{c}
r \\
1
\end{array}\right) \mu_{n} M^{*}+\left(\begin{array}{c}
r \\
2
\end{array}\right)\left(\mu_{n} M^{*}\right)^{2}+\left(\begin{array}{l}
r \\
3
\end{array}\right)\left(\mu_{n} M^{*}\right)^{3}+\cdots+\left(\begin{array}{l}
r \\
r
\end{array}\right)\left(\mu_{n} M^{*}\right)^{r}\right] \\
& +\sum_{j=0}^{r-1}\left(1+\mu_{n} M^{*}\right)^{j} \xi_{n} \\
\leqslant & \left(1+a_{n}^{r} \mu_{n}\right) d\left(x_{n}, p\right)+\sum_{j=0}^{r-1}\left(1+\mu_{n} M^{*}\right)^{j} \xi_{n} \\
\leqslant & \left(1+M_{1} \mu_{n}\right) d\left(x_{n}, p\right)+M_{2} \xi_{n},
\end{aligned}
$$

where $a_{n}^{r}=\left(\begin{array}{l}r \\ 1\end{array}\right) M^{*}+\left(\begin{array}{l}r \\ 2\end{array}\right)\left(M^{*}\right)^{2} \mu_{n}+\left(\begin{array}{l}r \\ 3\end{array}\right)\left(M^{*}\right)^{3}\left(\mu_{n}\right)^{2}+\cdots+\left(\begin{array}{l}r \\ r\end{array}\right)\left(M^{*}\right)^{r}\left(\mu_{n}\right)^{r-1}$, and it follows from condition (i) that there exist positive constants $M_{1}$ and $M_{2}$ such that $a_{n}^{r} \leqslant M_{1}, \sum_{j=0}^{r-1}\left(1+\mu_{n} M^{*}\right)^{j} \leqslant M_{2}$ for each $n \geqslant 1$. By Lemma 1.12, the inequality (2.4) implies that $\lim _{n \rightarrow \infty} d\left(x_{n}, p\right)$ exists for each $p \in F$.

Lemma 2.3. Let $\mathrm{K}$ be a nonempty closed and convex subset of a uniformly convex hyperbolic space $\mathrm{X}$ with monotone modulus of uniform convexity $\eta$. For $i \in \Re$, let $T_{i}: K \rightarrow X$ be a uniformly $L_{i}$-Lipschitzian and $\left(\left\{\mu_{n}^{i}\right\},\left\{\xi_{n}^{i}\right\}, \rho^{i}\right)-$ total quasi-asymptotically nonexpansive nonself-mapping, and $S_{i}: \mathrm{K} \rightarrow \mathrm{X}$ be a uniformly $\hat{\mathrm{L}}_{i}$-Lipschitzian and $\left(\left\{\hat{\mu}_{n}^{i}\right\},\left\{\hat{\xi}_{n}^{i}\right\}, \hat{\rho}^{i}\right)$-total asymptotically quasi-nonexpansive nonself-mapping, where constants $\mu_{n}^{i}, \xi_{n}^{i}, \rho^{i}, \hat{\mu}_{n}^{i}, \hat{\xi}_{n}^{i}, \hat{\rho}^{i}$ are the same as in Lemma 2.2. Suppose that $\mathrm{F}=\bigcap_{i=1}^{r}\left(\mathrm{~F}\left(\mathrm{~T}_{i}\right) \cap \mathrm{F}\left(\mathrm{S}_{i}\right)\right) \neq \emptyset$ and the conditions (i) and (ii) in Lemma 2.2 hold. Then, for $i \in \mathfrak{R}$ and the sequence $\left\{x_{n}\right\}$ in $(1.1)$, we have

$$
\lim _{n \rightarrow \infty} d\left(x_{n}, T_{i} x_{n}\right)=\lim _{n \rightarrow \infty} d\left(x_{n}, S_{i} x_{n}\right)=0 .
$$

Proof. From Lemma 2.2, it follows that $\lim _{n \rightarrow \infty} d\left(x_{n}, p\right)$ exists for each $p \in F$. Assume that

$$
\lim _{n \rightarrow \infty} d\left(x_{n}, p\right)=c>0 \text {. }
$$

Otherwise, the proof is trivial.

Since $\mu_{n} \rightarrow 0$ and $\xi_{n} \rightarrow 0$ as $n \rightarrow \infty$, taking limsup on both sides of inequalities (2.2) and (2.3), we have $\limsup _{n \rightarrow \infty} d\left(y_{n}, p\right) \leqslant c$ and $\limsup _{n \rightarrow \infty} d\left(y_{n+1}, p\right) \leqslant c$. Similarly, $\limsup _{n \rightarrow \infty} d\left(y_{n+r-2}, p\right) \leqslant c$, and so

$$
\limsup _{n \rightarrow \infty} d\left(y_{n+k-1}, p\right) \leqslant c, \quad \forall k=1,2, \cdots, r-1
$$


Since

$$
d\left(x_{n+1}, p\right) \leqslant\left(1+\mu_{n} M^{*}\right)^{r-1} d\left(y_{n}, p\right)+\sum_{j=0}^{r-2}\left(1+\mu_{n} M^{*}\right)^{j} \xi_{n}
$$

and taking lim inf on both sides of (2.6), we have

$$
\liminf _{n \rightarrow \infty} d\left(y_{n}, p\right) \geqslant c, \quad d\left(x_{n+1}, p\right) \leqslant\left(1+\mu_{n} M^{*}\right)^{r-k} d\left(y_{n+k-1}, p\right)+\sum_{j=0}^{r-k-1}\left(1+\mu_{n} M^{*}\right)^{j} \xi_{n}
$$

for all $k=2,3, \cdots, r-1$. Also setting lim inf on both side of the above estimate, then we have

$$
\liminf _{n \rightarrow \infty} d\left(y_{n+k-1}, p\right) \geqslant c, \quad \forall k=2,3, \cdots, r-1 .
$$

Thus, for every $k=1,2, \cdots, r-1$,

$$
\liminf _{n \rightarrow \infty} d\left(y_{n+k-1}, p\right) \geqslant c
$$

Combining (2.5) and (2.7), we have

$$
\lim _{n \rightarrow \infty} d\left(y_{n+k-1}, p\right)=c, \quad \forall k=1,2, \cdots, r-1 .
$$

For $k=1$ in (2.8), and combining (2.2), we get

$$
\lim _{n \rightarrow \infty} d\left(W\left(T_{r}\left(P T_{r}\right)^{n-1} \chi_{n}, W\left(x_{n}, S_{r}\left(P S_{r}\right)^{n-1} \chi_{n}, \theta_{r n}\right), \alpha_{r n}\right), p\right)=c .
$$

Moreover,

$$
\begin{aligned}
d\left(W\left(x_{n}, S_{r}\left(P S_{r}\right)^{n-1} x_{n}, \theta_{r n}\right), p\right) & \leqslant \theta_{r n} d\left(x_{n}, p\right)+\left(1-\theta_{r n}\right) d\left(S_{r}\left(P S_{r}\right)^{n-1} x_{n}, p\right) \\
& \leqslant \theta_{r n} d\left(x_{n}, p\right)+\left(1-\theta_{r n}\right)\left[\left(1+\mu_{n} M^{*}\right) d\left(x_{n}, p\right)+\xi_{n}\right] \\
& \leqslant\left(1+\mu_{n} M^{*}\right) d\left(x_{n}, p\right)+\xi_{n}
\end{aligned}
$$

which implies that

$$
\limsup _{n \rightarrow \infty} d\left(W\left(x_{n}, S_{r}\left(P S_{r}\right)^{n-1} x_{n}, \theta_{r n}\right), p\right) \leqslant c
$$

Obviously,

$$
\limsup _{n \rightarrow \infty} d\left(T_{r}\left(P T_{r}\right)^{n-1} x_{n}, p\right) \leqslant c .
$$

It follows from (2.9)-(2.11) and Lemma 1.11 that

$$
\lim _{n \rightarrow \infty} d\left(T_{r}\left(P T_{r}\right)^{n-1} x_{n}, W\left(x_{n}, S_{r}\left(P S_{r}\right)^{n-1} x_{n}, \theta_{r n}\right)\right)=0 .
$$

Again, for $k=2,3, \cdots, r-1,(2.8)$ is rewritten as

$$
\begin{aligned}
\lim _{n \rightarrow \infty} & d\left(P W \left(T_{r-(k-1)}\left(P_{r-(k-1)}\right)^{n-1} y_{n+k-2}\right.\right. \\
& \left.\left.W\left(y_{n+k-2}, S_{r-(k-1)}\left(P S_{r-(k-1)}\right)^{n-1} y_{n+k-2}, \theta_{(r-k+1) n}\right), \alpha_{(r-k+1) n}\right), p\right)=c .
\end{aligned}
$$

In a similar way to (2.8), we have

$$
\begin{aligned}
\lim _{n \rightarrow \infty} & d\left(W \left(T_{r-(k-1)}\left(P T_{r-(k-1)}\right)^{n-1} y_{n+k-2}\right.\right. \\
& \left.\left.W\left(y_{n+k-2}, S_{r-(k-1)}\left(P S_{r-(k-1)}\right)^{n-1} y_{n+k-2}, \theta_{(r-k+1) n}\right), \alpha_{(r-k+1) n}\right), p\right)=c .
\end{aligned}
$$


By (2.5) and the following inequality

$$
\begin{aligned}
d & \left(W\left(y_{n+k-2}, S_{r-(k-1)}\left(P S_{r-(k-1)}\right)^{n-1} y_{n+k-2}, \theta_{(r-k+1) n}\right), p\right) \\
& \leqslant \theta_{(r-k+1) n} d\left(y_{n+k-2}, p\right)+\left(1-\theta_{(r-k+1) n}\right) d\left(S_{r-(k-1)}\left(P S_{r-(k-1)}\right)^{n-1} y_{n+k-2}, p\right) \\
& \leqslant \theta_{(r-k+1) n} d\left(y_{n+k-2}, p\right)+\left(1-\theta_{(r-k+1) n}\right)\left[\left(1+\mu_{n} M^{*}\right) d\left(y_{n+k-2}, p\right)+\xi_{n}\right] \\
& \leqslant\left(1+\mu_{n} M^{*}\right) d\left(y_{n+k-2}, p\right)+\xi_{n},
\end{aligned}
$$

now we know that

$$
\limsup _{n \rightarrow \infty} d\left(W\left(y_{n+k-2}, S_{r-(k-1)}\left(P S_{r-(k-1)}\right)^{n-1} y_{n+k-2,}, \theta_{(r-k+1) n}\right), p\right) \leqslant c .
$$

Further,

$$
\limsup _{n \rightarrow \infty} d\left(T_{r-(k-1)}\left(P T_{r-(k-1)}\right)^{n-1} y_{n+k-2,} p\right) \leqslant c, \quad \forall k=2,3, \cdots, r-1 .
$$

From (2.13)-(2.15) and Lemma 1.11, it follows that

$$
\begin{aligned}
\lim _{n \rightarrow \infty} & d\left(T_{r-(k-1)}\left(P T_{r-(k-1)}\right)^{n-1} y_{n+k-2},\right. \\
& \left.W\left(y_{n+k-2}, S_{r-(k-1)}\left(P S_{r-(k-1)}\right)^{n-1} y_{n+k-2}, \theta_{(r-k+1) n}\right)\right)=0
\end{aligned}
$$

for $k=2,3, \cdots, r-1$. When $k=r$, we get

$$
\lim _{n \rightarrow \infty} d\left(x_{n+1}, p\right)=\lim _{n \rightarrow \infty} d\left(P W\left(T_{1}\left(P T_{1}\right)^{n-1} y_{n+r-2}, W\left(y_{n+r-2}, S_{1}\left(P S_{1}\right)^{n-1} y_{n+r-2}, \theta_{1 n}\right), \alpha_{1 n}\right), p\right)=c
$$

This implies that

$$
\lim _{n \rightarrow \infty} d\left(W\left(T_{1}\left(P T_{1}\right)^{n-1} y_{n+r-2}, W\left(y_{n+r-2}, S_{1}\left(P S_{1}\right)^{n-1} y_{n+r-2}, \theta_{1 n}\right), \alpha_{1 n}\right), p\right)=c .
$$

By (2.5), the following estimate

$$
\begin{aligned}
d\left(W\left(y_{n+r-2}, S_{1}\left(P S_{1}\right)^{n-1} y_{n+r-2}, \theta_{1 n}\right), p\right) & \leqslant \theta_{1 n} d\left(y_{n+r-2}, p\right)+\left(1-\theta_{1 n}\right) d\left(S_{1}\left(P S_{1}\right)^{n-1} y_{n+r-2}, p\right) \\
& \leqslant \theta_{1 n} d\left(y_{n+r-2}, p\right)+\left(1-\theta_{1 n}\right)\left[\left(1+\mu_{n} M^{*}\right) d\left(y_{n+r-2}, p\right)+\xi_{n}\right] \\
& \leqslant\left(1+\mu_{n} M^{*}\right) d\left(y_{n+r-2}, p\right)+\xi_{n}
\end{aligned}
$$

implies that

$$
\limsup _{n \rightarrow \infty} d\left(W\left(y_{n+r-2}, S_{1}\left(P S_{1}\right)^{n-1} y_{n+r-2}, \theta_{1 n}\right), p\right) \leqslant c .
$$

Also,

$$
\limsup _{n \rightarrow \infty} d\left(T_{1}\left(P T_{1}\right)^{n-1} y_{n+r-2}, p\right) \leqslant c .
$$

Hence, (2.17)-(2.19) and Lemma 1.11 imply that

$$
\lim _{n \rightarrow \infty} d\left(T_{1}\left(P T_{1}\right)^{n-1} y_{n+r-2}, W\left(y_{n+r-2}, S_{1}\left(P S_{1}\right)^{n-1} y_{n+r-2}, \theta_{1 n}\right)\right)=0,
$$

and

$$
\begin{aligned}
& d\left(W\left(T_{1}\left(P T_{1}\right)^{n-1} y_{n+r-2}, W\left(y_{n+r-2}, S_{1}\left(P S_{1}\right)^{n-1} y_{n+r-2}, \theta_{1 n}\right), \alpha_{1 n}\right), T_{1}\left(P T_{1}\right)^{n-1} y_{n+r-2}\right) \\
& \quad \leqslant\left(1-\alpha_{1 n}\right) d\left(W\left(y_{n+r-2}, S_{1}\left(P S_{1}\right)^{n-1} y_{n+r-2}, \theta_{1 n}\right), T_{1}\left(P T_{1}\right)^{n-1} y_{n+r-2}\right)
\end{aligned}
$$




$$
+\alpha_{1 n} d\left(T_{1}\left(P T_{1}\right)^{n-1} y_{n+r-2}, T_{1}\left(P T_{1}\right)^{n-1} y_{n+r-2}\right) .
$$

Based on (2.20), this implies

$$
\lim _{n \rightarrow \infty} d\left(W\left(T_{1}\left(P T_{1}\right)^{n-1} y_{n+r-2}, W\left(y_{n+r-2}, S_{1}\left(P S_{1}\right)^{n-1} y_{n+r-2}, \theta_{1 n}\right), \alpha_{1 n}\right), T_{1}\left(P T_{1}\right)^{n-1} y_{n+r-2}\right)=0,
$$

and so

$$
\lim _{n \rightarrow \infty} d\left(x_{n+1},\left(P T_{1}\right)^{n} y_{n+r-2}\right)=0 .
$$

In addition, it follows from (2.1) that

$$
\begin{aligned}
d & \left(W\left(T_{1}\left(P T_{1}\right)^{n-1} y_{n+r-2}, W\left(y_{n+r-2}, S_{1}\left(P S_{1}\right)^{n-1} y_{n+r-2}, \theta_{1 n}\right), \alpha_{1 n}\right), p\right) \\
\leqslant & \alpha_{1 n} d\left(T_{1}\left(P T_{1}\right)^{n-1} y_{n+r-2}, p\right)+\left(1-\alpha_{1 n}\right) d\left(W\left(y_{n+r-2}, S_{1}\left(P S_{1}\right)^{n-1} y_{n+r-2}, \theta_{1 n}\right), p\right) \\
\leqslant & \alpha_{1 n} d\left(W\left(T_{1}\left(P T_{1}\right)^{n-1} y_{n+r-2}, W\left(y_{n+r-2}, S_{1}\left(P S_{1}\right)^{n-1} y_{n+r-2}, \theta_{1 n}\right), \alpha_{1 n}\right), p\right) \\
& +\alpha_{1 n} d\left(W\left(T_{1}\left(P T_{1}\right)^{n-1} y_{n+r-2}, W\left(y_{n+r-2}, S_{1}\left(P S_{1}\right)^{n-1} y_{n+r-2}, \theta_{1 n}\right), \alpha_{1 n}\right), T_{1}\left(P T_{1}\right)^{n-1} y_{n+r-2}\right) \\
& +\left(1-\alpha_{1 n}\right) d\left(W\left(y_{n+r-2}, S_{1}\left(P S_{1}\right)^{n-1} y_{n+r-2}, \theta_{1 n}\right), p\right) \\
\leqslant & \frac{\alpha_{1 n}}{1-\alpha_{1 n}} d\left(W\left(T_{1}\left(P T_{1}\right)^{n-1} y_{n+r-2}, W\left(y_{n+r-2}, S_{1}\left(P S_{1}\right)^{n-1} y_{n+r-2}, \theta_{1 n}\right), \alpha_{1 n}\right), T_{1}\left(P T_{1}\right)^{n-1} y_{n+r-2}\right) \\
& +d\left(W\left(y_{n+r-2}, S_{1}\left(P S_{1}\right)^{n-1} y_{n+r-2}, \theta_{1 n}\right), p\right) \\
\leqslant & \frac{b}{1-b} d\left(W\left(T_{1}\left(P T_{1}\right)^{n-1} y_{n+r-2}, W\left(y_{n+r-2}, S_{1}\left(P S_{1}\right)^{n-1} y_{n+r-2}, \theta_{1 n}\right), \alpha_{1 n}\right), T_{1}\left(P T_{1}\right)^{n-1} y_{n+r-2}\right) \\
& +d\left(W\left(y_{n+r-2}, S_{1}\left(P S_{1}\right)^{n-1} y_{n+r-2}, \theta_{1 n}\right), p\right) .
\end{aligned}
$$

Taking lim inf on both sides of the estimate (2.22), and from (2.17) and (2.21), we have

$$
\liminf _{n \rightarrow \infty} d\left(W\left(y_{n+r-2}, S_{1}\left(P S_{1}\right)^{n-1} y_{n+r-2}, \theta_{1 n}\right), p\right) \geqslant c .
$$

Combining (2.18) and (2.23), we get

$$
\lim _{n \rightarrow \infty} d\left(W\left(y_{n+r-2}, S_{1}\left(P S_{1}\right)^{n-1} y_{n+r-2}, \theta_{1 n}\right), p\right)=c .
$$

By Lemma 1.11 and (2.24), we have

$$
\lim _{n \rightarrow \infty} d\left(y_{n+r-2}, S_{1}\left(P S_{1}\right)^{n-1} y_{n+r-2}\right)=0 .
$$

In a similar way, for $k=2,3, \cdots, r-1$, we compute

$$
\begin{aligned}
& d\left(W\left(T_{r-(k-1)}\left(P T_{r-(k-1)}\right)^{n-1} y_{n+k-2}, W\left(y_{n+k-2}, S_{r-(k-1)}\left(P S_{r-(k-1)}\right)^{n-1} y_{n+k-2}, \theta_{(r-k+1) n}\right), \alpha_{(r-k+1) n}\right),\right. \\
& \left.\quad T_{r-(k-1)}\left(P T_{r-(k-1)}\right)^{n-1} y_{n+k-2}\right) \\
& \leqslant\left(1-\alpha_{(r-k+1) n}\right) d\left(W\left(y_{n+k-2}, S_{r-(k-1)}\left(P S_{r-(k-1)}\right)^{n-1} y_{n+k-2}, \theta_{(r-k+1) n}\right),\right. \\
& \left.\quad T_{r-(k-1)}\left(P T_{r-(k-1)}\right)^{n-1} y_{n+k-2}\right) \\
& \quad+\alpha_{(r-k+1) n} d\left(T_{r-(k-1)}\left(P T_{r-(k-1)}\right)^{n-1} y_{n+k-2}, T_{r-(k-1)}\left(P T_{r-(k-1)}\right)^{n-1} y_{n+k-2}\right) .
\end{aligned}
$$

Utilizing (2.16), we have

$$
\begin{gathered}
\lim _{n \rightarrow \infty} d\left(W \left(T_{r-(k-1)}\left(P T_{r-(k-1)}\right)^{n-1} y_{n+k-2}, W\left(y_{n+k-2}, S_{r-(k-1)}\left(P S_{r-(k-1)}\right)^{n-1} y_{n+k-2,}\right.\right.\right. \\
\left.\left.\left.\theta_{(r-k+1) n}\right), \alpha_{(r-k+1) n}\right), T_{r-(k-1)}\left(P T_{r-(k-1)}\right)^{n-1} y_{n+k-2}\right)=0, \quad \forall k=2,3, \cdots, r-1
\end{gathered}
$$


and so

$$
\lim _{n \rightarrow \infty} d\left(y_{n+k-1},\left(P T_{r-(k-1)}\right)^{n} y_{n+k-2}\right)=0, \quad \forall k=2,3, \cdots, r-1 .
$$

For $k=1$, we calculate

$$
\begin{aligned}
& d\left(W\left(T_{r}\left(P T_{r}\right)^{n-1} x_{n}, W\left(x_{n}, S_{r}\left(P S_{r}\right)^{n-1} x_{n}, \theta_{r n}\right), \alpha_{r n}\right), T_{r}\left(P T_{r}\right)^{n-1} x_{n}\right) \\
& \quad \leqslant \alpha_{r n} d\left(T_{r}\left(P T_{r}\right)^{n-1} x_{n}, T_{r}\left(P T_{r}\right)^{n-1} x_{n}\right)+\left(1-\alpha_{r n}\right) d\left(W\left(x_{n}, S_{r}\left(P S_{r}\right)^{n-1} x_{n}, \theta_{r n}\right), T_{r}\left(P T_{r}\right)^{n-1} x_{n}\right) .
\end{aligned}
$$

Now, using (2.12), we have

$$
\lim _{n \rightarrow \infty} d\left(W\left(T_{r}\left(P T_{r}\right)^{n-1} x_{n}, W\left(x_{n}, S_{r}\left(P S_{r}\right)^{n-1} x_{n}, \theta_{r n}\right), \alpha_{r n}\right), T_{r}\left(P T_{r}\right)^{n-1} x_{n}\right)=0,
$$

and so

$$
\lim _{n \rightarrow \infty} d\left(y_{n},\left(P T_{r}\right)^{n} x_{n}\right)=0
$$

Reasoning as above, we get

$$
\begin{aligned}
& d\left(W\left(T_{r}\left(P T_{r}\right)^{n-1} x_{n}, W\left(x_{n}, S_{r}\left(P S_{r}\right)^{n-1} x_{n}, \theta_{r n}\right), \alpha_{r n}\right), p\right) \\
& \leqslant \frac{b}{1-b} d\left(T_{r}\left(P T_{r}\right)^{n-1} x_{n}, W\left(T_{r}\left(P T_{r}\right)^{n-1} x_{n}, W\left(x_{n}, S_{r}\left(P S_{r}\right)^{n-1} x_{n}, \theta_{r n}\right), \alpha_{r n}\right)\right) \\
& \quad+d\left(W\left(x_{n}, S_{r}\left(P S_{r}\right)^{n-1} x_{n}, \theta_{r n}\right), p\right) .
\end{aligned}
$$

Setting lim inf on both sides of the estimate (2.28) and utilizing (2.9) and (2.27), we know

$$
\liminf _{n \rightarrow \infty} d\left(W\left(x_{n}, S_{r}\left(P S_{r}\right)^{n-1} x_{n}, \theta_{r n}\right), p\right) \geqslant c .
$$

Inequalities (2.10) and (2.29) collectively imply that

$$
\lim _{n \rightarrow \infty} d\left(W\left(x_{n}, S_{r}\left(P S_{r}\right)^{n-1} x_{n}, \theta_{r n}\right), p\right)=c .
$$

Consequently, Lemma 1.11 and (2.30) imply that

$$
\lim _{n \rightarrow \infty} d\left(x_{n}, S_{r}\left(P S_{r}\right)^{n-1} x_{n}\right)=0
$$

Note that

$$
\begin{aligned}
d\left(x_{n}, T_{r}\left(P T_{r}\right)^{n-1} x_{n}\right) \leqslant & d\left(x_{n}, W\left(T_{r}\left(P T_{r}\right)^{n-1} x_{n}, W\left(x_{n}, S_{r}\left(P S_{r}\right)^{n-1} x_{n}, \theta_{r n}\right), \alpha_{r n}\right)\right) \\
& +d\left(W\left(T_{r}\left(P T_{r}\right)^{n-1} x_{n}, W\left(x_{n}, S_{r}\left(P S_{r}\right)^{n-1} x_{n}, \theta_{r n}\right), \alpha_{r n}\right), T_{r}\left(P T_{r}\right)^{n-1} x_{n}\right) \\
\leqslant & \alpha_{r n} d\left(x_{n}, T_{r}\left(P T_{r}\right)^{n-1} x_{n}\right)+\left(1-\alpha_{r n}\right) d\left(W\left(x_{n}, S_{r}\left(P S_{r}\right)^{n-1} x_{n}, \theta_{r n}\right), x_{n}\right) \\
& +d\left(W\left(T_{r}\left(P T_{r}\right)^{n-1} x_{n}, W\left(x_{n}, S_{r}\left(P S_{r}\right)^{n-1} x_{n}, \theta_{r n}\right), \alpha_{r n}\right), T_{r}\left(P T_{r}\right)^{n-1} x_{n}\right) \\
\leqslant & \left(1-\theta_{r n}\right) d\left(x_{n}, S_{r}\left(P S_{r}\right)^{n-1} x_{n}\right) \\
& +\frac{1}{1-\alpha_{r n}} d\left(W\left(T_{r}\left(P T_{r}\right)^{n-1} x_{n}, W\left(x_{n}, S_{r}\left(P S_{r}\right)^{n-1} x_{n}, \theta_{r n}\right), \alpha_{r n}\right), T_{r}\left(P T_{r}\right)^{n-1} x_{n}\right) \\
\leqslant & \frac{1-a}{1-b} d\left(x_{n}, S_{r}\left(P S_{r}\right)^{n-1} x_{n}\right) \\
& +\frac{1}{1-b} d\left(W\left(T_{r}\left(P T_{r}\right)^{n-1} x_{n}, W\left(x_{n}, S_{r}\left(P S_{r}\right)^{n-1} x_{n}, \theta_{r n}\right), \alpha_{r n}\right), T_{r}\left(P T_{r}\right)^{n-1} x_{n}\right) .
\end{aligned}
$$


From (2.27) and (2.31), we have

$$
\lim _{n \rightarrow \infty} d\left(x_{n}, T_{r}\left(P T_{r}\right)^{n-1} x_{n}\right)=0
$$

Moreover

$$
\begin{aligned}
& d\left(x_{n}, W\left(T_{r}\left(P T_{r}\right)^{n-1} x_{n}, W\left(x_{n}, S_{r}\left(P S_{r}\right)^{n-1} x_{n}, \theta_{r n}\right), \alpha_{r n}\right)\right) \\
& \quad \leqslant \alpha_{r n} d\left(x_{n}, T_{r}\left(P T_{r}\right)^{n-1} x_{n}\right)+\left(1-\alpha_{r n}\right) d\left(x_{n}, W\left(x_{n}, S_{r}\left(P S_{r}\right)^{n-1} x_{n}, \theta_{r n}\right)\right) \\
& \quad \leqslant \alpha_{r n} d\left(x_{n}, T_{r}\left(P T_{r}\right)^{n-1} x_{n}\right)+\left(1-\alpha_{r n}-\beta_{r n}\right) d\left(x_{n}, S_{r}\left(P S_{r}\right)^{n-1} x_{n}\right) \\
& \quad \leqslant b d\left(x_{n}, T_{r}\left(P T_{r}\right)^{n-1} x_{n}\right)+(1-2 a) d\left(x_{n}, S_{r}\left(P S_{r}\right)^{n-1} x_{n}\right)
\end{aligned}
$$

By (2.31) and (2.32), we have

$$
\lim _{n \rightarrow \infty} d\left(x_{n}, W\left(T_{r}\left(P T_{r}\right)^{n-1} x_{n}, W\left(x_{n}, S_{r}\left(P S_{r}\right)^{n-1} x_{n}, \theta_{r n}\right), \alpha_{r n}\right)\right)=0 .
$$

Since

$$
\begin{aligned}
d\left(x_{n}, y_{n}\right) & =d\left(P x_{n}, P\left(W\left(T_{r}\left(P T_{r}\right)^{n-1} x_{n}, W\left(x_{n}, S_{r}\left(P S_{r}\right)^{n-1} x_{n}, \theta_{r n}\right), \alpha_{r n}\right)\right)\right. \\
& \leqslant d\left(x_{n}, W\left(T_{r}\left(P T_{r}\right)^{n-1} x_{n}, W\left(x_{n}, S_{r}\left(P S_{r}\right)^{n-1} x_{n}, \theta_{r n}\right), \alpha_{r n}\right)\right)
\end{aligned}
$$

we have

$$
\lim _{n \rightarrow \infty} d\left(x_{n}, y_{n}\right)=0
$$

Similarly, we have

$$
\begin{aligned}
d\left(y_{n+k-1}, p\right) \leqslant & d\left(W \left(T_{r-(k-1)}\left(P T_{r-(k-1)}\right)^{n-1} y_{n+k-2,}\right.\right. \\
& \left.\left.W\left(y_{n+k-2}, S_{r-(k-1)}\left(P S_{r-(k-1)}\right)^{n-1} y_{n+k-2,}, \theta_{(r-k+1) n}\right), \alpha_{(r-k+1) n}\right), p\right) \\
\leqslant & d\left(W\left(y_{n+k-2}, S_{r-(k-1)}\left(P S_{r-(k-1)}\right)^{n-1} y_{n+k-2}, \theta_{(r-k+1) n}\right), p\right) \\
+ & \frac{b}{1-b} d\left(T_{r-(k-1)}\left(P T_{r-(k-1)}\right)^{n-1} y_{n+k-2}, W\left(T_{r-(k-1)}\left(P T_{r-(k-1)}\right)^{n-1} y_{n+k-2},\right.\right. \\
& \left.\left.W\left(y_{n+k-2}, S_{r-(k-1)}\left(P S_{r-(k-1)}\right)^{n-1} y_{n+k-2,}, \theta_{(r-k+1) n}\right), \alpha_{(r-k+1) n}\right)\right) .
\end{aligned}
$$

Now, Utilizing (2.7) and (2.25), we get

$$
\liminf _{n \rightarrow \infty} d\left(W\left(y_{n+k-2}, S_{r-(k-1)}\left(P S_{r-(k-1)}\right)^{n-1} y_{n+k-2}, \theta_{(r-k+1) n}\right), p\right) \geqslant c .
$$

Thus, (2.14) and (2.34) imply

$$
\lim _{n \rightarrow \infty} d\left(W\left(y_{n+k-2}, S_{r-(k-1)}\left(P S_{r-(k-1)}\right)^{n-1} y_{n+k-2}, \theta_{(r-k+1) n}\right), p\right)=c,
$$

and by Lemma 1.11, we know that

$$
\lim _{n \rightarrow \infty} d\left(y_{n+k-2,}, S_{r-(k-1)}\left(P S_{r-(k-1)}\right)^{n-1} y_{n+k-2}\right)=0 .
$$

for all $k=2,3, \cdots, r-1$. Also,

$$
\begin{aligned}
& d\left(y_{n+k-2}, T_{r-(k-1)}\left(P T_{r-(k-1)}\right)^{n-1} y_{n+k-2}\right) \\
& \leqslant \\
& \quad d\left(y_{n+k-2}, W\left(T_{r-(k-1)}\left(P T_{r-(k-1)}\right)^{n-1} y_{n+k-2},\right.\right. \\
& \left.\left.\quad W\left(y_{n+k-2}, S_{r-(k-1)}\left(P S_{r-(k-1)}\right)^{n-1} y_{n+k-2}, \theta_{(r-k+1) n}\right), \alpha_{(r-k+1) n}\right)\right) \\
& +d\left(W \left(T_{r-(k-1)}\left(P T_{r-(k-1)}\right)^{n-1} y_{n+k-2},\right.\right.
\end{aligned}
$$




$$
\begin{aligned}
& \left.\left.W\left(y_{n+k-2}, S_{r-(k-1)}\left(P S_{r-(k-1)}\right)^{n-1} y_{n+k-2}, \theta_{(r-k+1) n}\right), \alpha_{(r-k+1) n}\right), T_{r-(k-1)}\left(P T_{r-(k-1)}\right)^{n-1} y_{n+k-2}\right) \\
\leqslant & \alpha_{(r-k+1) n} d\left(y_{n+k-2}, T_{r-(k-1)}\left(P T_{r-(k-1)}\right)^{n-1} y_{n+k-2}\right) \\
+ & \left(1-\alpha_{(r-k+1) n}\right) d\left(y_{n+k-2}, W\left(y_{n+k-2}, S_{r-(k-1)}\left(P S_{r-(k-1)}\right)^{n-1} y_{n+k-2}, \theta_{(r-k+1) n}\right)\right) \\
+ & d\left(W \left(T_{r-(k-1)}\left(P T_{r-(k-1)}\right)^{n-1} y_{n+k-2}\right.\right. \\
& \left.\left.W\left(y_{n+k-2}, S_{r-(k-1)}\left(P S_{r-(k-1)}\right)^{n-1} y_{n+k-2}, \theta_{(r-k+1) n}\right), \alpha_{(r-k+1) n}\right), T_{r-(k-1)}\left(P T_{r-(k-1)}\right)^{n-1} y_{n+k-2}\right) \\
\leqslant & \left(1-\theta_{(r-k+1) n}\right) d\left(y_{n+k-2}, S_{r-(k-1)}\left(P S_{r-(k-1)}\right)^{n-1} y_{n+k-2}\right) \\
+ & \frac{1}{1-\alpha_{(r-k+1) n}} d\left(W \left(T_{r-(k-1)}\left(P T_{r-(k-1)}\right)^{n-1} y_{n+k-2},\right.\right. \\
& \left.\left.W\left(y_{n+k-2}, S_{r-(k-1)}\left(P S_{r-(k-1)}\right)^{n-1} y_{n+k-2}, \theta_{(r-k+1) n}\right), \alpha_{(r-k+1) n}\right), T_{r-(k-1)}\left(P T_{r-(k-1)}\right)^{n-1} y_{n+k-2}\right) \\
\leqslant & \frac{1-2 a}{1-b} d\left(y_{n+k-2}, S_{r-(k-1)}\left(P S_{r-(k-1)}\right)^{n-1} y_{n+k-2}\right) \\
+ & \frac{1}{1-b} d\left(W \left(T_{r-(k-1)}\left(P T_{r-(k-1)}\right)^{n-1} y_{n+k-2},\right.\right. \\
& \left.\left.W\left(y_{n+k-2}, S_{r-(k-1)}\left(P S_{r-(k-1)}\right)^{n-1} y_{n+k-2}, \theta_{(r-k+1) n}\right), \alpha_{(r-k+1) n}\right), T_{r-(k-1)}\left(P T_{r-(k-1)}\right)^{n-1} y_{n+k-2}\right) .
\end{aligned}
$$

Now, it follows from (2.25) and (2.35) that for all $k=2,3, \cdots, r-1$,

$$
\lim _{n \rightarrow \infty} d\left(y_{n+k-2}, T_{r-(k-1)}\left(P T_{r-(k-1)}\right)^{n-1} y_{n+k-2}\right)=0 .
$$

For $k=2,3, \cdots, r-1$, we have

$$
\begin{aligned}
d\left(y_{n+k-2}, y_{n+k-1}\right) & \leqslant d\left(y_{n+k-2},\left(P T_{r-(k-1)}\right)^{n} y_{n+k-2}\right)+d\left(\left(P T_{r-(k-1)}\right)^{n} y_{n+k-2}, y_{n+k-1}\right) \\
& =d\left(P y_{n+k-2},\left(P T_{r-(k-1)}\right)^{n} y_{n+k-2}\right)+d\left(\left(P T_{r-(k-1)}\right)^{n} y_{n+k-2}, y_{n+k-1}\right) \\
& \leqslant d\left(y_{n+k-2}, T_{r-(k-1)}\left(P T_{r-(k-1)}\right)^{n-1} y_{n+k-2}\right)+d\left(\left(P T_{r-(k-1)}\right)^{n} y_{n+k-2}, y_{n+k-1}\right)
\end{aligned}
$$

Hence, (2.26) and (2.36) imply that

$$
\lim _{n \rightarrow \infty} d\left(y_{n+k-2}, y_{n+k-1}\right)=0
$$

Additionally,

$$
d\left(x_{n}, y_{n+k-1}\right) \leqslant d\left(x_{n}, y_{n}\right)+d\left(y_{n}, y_{n+1}\right)+\cdots+d\left(y_{n+k-2}, y_{n+k-1}\right) .
$$

By (2.33) and (2.37), we have

$$
\lim _{n \rightarrow \infty} d\left(x_{n}, y_{n+k-1}\right)=0, \quad \forall k=1,2, \cdots, r-1 .
$$

Let $L=\max _{i \in \mathfrak{R}}\left\{L_{i}, \hat{L}_{i}\right\}$, where $L_{i}$ and $\hat{L}_{i}$ are Lipschitz constants for $T_{i}$ and $S_{i}$ for $i \in \Re$, respectively. Since each $T_{i}$ is uniformly L-Lipschitzian for $i \in \mathfrak{R}$, we have

$$
\begin{aligned}
d\left(x_{n}, T_{i}\left(P T_{i}\right)^{n-1} x_{n}\right) \leqslant & d\left(x_{n}, y_{n+r-i-1}\right)+d\left(y_{n+r-i-1}, T_{i}\left(P T_{i}\right)^{n-1} x_{n}\right) \\
\leqslant & d\left(x_{n}, y_{n+r-i-1}\right)+d\left(y_{n+r-i-1}, T_{i}\left(P T_{i}\right)^{n-1} y_{n+r-i-1}\right) \\
& +d\left(T_{i}\left(P T_{i}\right)^{n-1} y_{n+r-i-1}, T_{i}\left(P T_{i}\right)^{n-1} x_{n}\right) \\
\leqslant & (1+L) d\left(x_{n}, y_{n+r-i-1}\right)+d\left(y_{n+r-i-1}, T_{i}\left(P T_{i}\right)^{n-1} y_{n+r-i-1}\right)
\end{aligned}
$$

for $1 \leqslant i \leqslant r-1$. It follows from (2.36) and (2.38) that

$$
\lim _{n \rightarrow \infty} d\left(x_{n}, T_{i}\left(P T_{i}\right)^{n-1} x_{n}\right)=0, \quad \forall 1 \leqslant i \leqslant r-1 .
$$


Moreover,

$$
\begin{aligned}
& d\left(x_{n+1}, T_{i} x_{n+1}\right) \\
& \leqslant d\left(x_{n+1}, T_{i}\left(P T_{i}\right)^{n} x_{n+1}\right)+d\left(T_{i}\left(P T_{i}\right)^{n} x_{n+1}, T_{i}\left(P T_{i}\right)^{n} y_{n+r-i-1}\right)+d\left(T_{i}\left(P T_{i}\right)^{n} y_{n+r-i-1}, T_{i} x_{n+1}\right) \\
& \leqslant d\left(x_{n+1}, T_{i}\left(P T_{i}\right)^{n} x_{n+1}\right)+\operatorname{Ld}\left(x_{n+1}, y_{n+r-i-1}\right)+\operatorname{Ld}\left(\left(P T_{i}\right)^{n} y_{n+r-i-1}, x_{n+1}\right) \\
& \leqslant d\left(x_{n+1}, T_{i}\left(P T_{i}\right)^{n} x_{n+1}\right)+2 \operatorname{Ld}\left(x_{n+1}, y_{n+r-i-1}\right)+\operatorname{Ld}\left(\left(P T_{i}\right)^{n} y_{n+r-i-1}, y_{n+r-i-1}\right) \\
& \leqslant d\left(x_{n+1}, T_{i}\left(P T_{i}\right)^{n} x_{n+1}\right)+2 L d\left(x_{n+1}, y_{n+r-i-1}\right)+L d\left(P T_{i}\left(P T_{i}\right)^{n-1} y_{n+r-i-1}, P y_{n+r-i-1}\right) \\
& \leqslant d\left(x_{n+1}, T_{i}\left(P T_{i}\right)^{n} x_{n+1}\right)+2 L d\left(x_{n+1}, y_{n+r-i-1}\right)+\operatorname{Ld}\left(T_{i}\left(P T_{i}\right)^{n-1} y_{n+r-i-1}, y_{n+r-i-1}\right) .
\end{aligned}
$$

Thus, (2.36), (2.38), and (2.39) (or (2.32)) imply that $d\left(x_{n+1}, T_{i} x_{n+1}\right) \rightarrow 0$ as $n \rightarrow \infty$ and so

$$
\lim _{n \rightarrow \infty} d\left(x_{n}, T_{i} x_{n}\right)=0, \quad \forall 1 \leqslant i \leqslant r .
$$

Similarly, we have

$$
\lim _{n \rightarrow \infty} d\left(x_{n}, S_{i} x_{n}\right)=0, \quad \forall 1 \leqslant i \leqslant r .
$$

This completes the proof.

Now we are in a position to give $\Delta$-convergence results of this paper.

Theorem 2.4. Let $\mathrm{K}$ be a nonempty closed and convex subset of a complete uniformly convex hyperbolic space $\mathrm{X}$ with monotone modulus of uniform convexity $\eta$. For $i \in \mathfrak{R}=\{1,2,3, \cdots, r\}$, let $\mathrm{T}_{i}$ and $\mathrm{S}_{i}$ be the same in Lemma 2.3. Assume that $\mathrm{F}=\bigcap_{i=1}^{r}\left(\mathrm{~F}\left(\mathrm{~T}_{i}\right) \cap \mathrm{F}\left(\mathrm{S}_{i}\right)\right) \neq \emptyset$ and for $i \in \mathfrak{R}$, the following conditions hold:

(i) $\sum_{n=1}^{\infty} \mu_{n}^{i}<+\infty, \sum_{n=1}^{\infty} \hat{\mu}_{n}^{i}<+\infty, \sum_{n=1}^{\infty} \xi_{n}^{i}<+\infty, \sum_{n=1}^{\infty} \hat{\xi}_{n}^{i}<+\infty$.

(ii) There exist constants $M_{i}>0$ and $\hat{M}_{i}>0$ such that

$$
\rho^{i}(r) \leqslant M_{i} r, \quad \hat{\rho}^{i}(r) \leqslant \hat{M}_{i} r, \quad \forall r>0 .
$$

Then the sequence $\left\{x_{n}\right\}$ defined in (1.1) $\Delta$-converges to a common fixed point $p \in F$.

Proof. Since it follows from Lemma 2.2 that the sequence $\left\{x_{n}\right\}$ defined in (1.1) is bounded, Lemma 1.10 asserts that $\left\{x_{n}\right\}$ has a unique asymptotic center in K. That is, $A_{K}\left(\left\{x_{n}\right\}\right)=\{x\}$. Let $\left\{v_{n}\right\}$ be any subsequence of $\left\{x_{n}\right\}$ such that $A_{K}\left(\left\{v_{n}\right\}\right)=\{v\}$. Then, by Lemma 2.3, we have

$$
\lim _{n \rightarrow \infty} \mathrm{d}\left(v_{n}, \mathrm{~T}_{i} v_{n}\right)=\lim _{n \rightarrow \infty} \mathrm{d}\left(v_{n}, S_{i} v_{n}\right)=0, \quad \forall i \in \Re .
$$

By Lemma 1.13, we know that $v$ is the common fixed point of $\left\{T_{i}\right\}_{i \in \mathfrak{R}}$ and $\left\{S_{i}\right\}_{i \in \Re}$, i.e., $v \in F$.

Next, we claim that the common fixed point $v$ is the unique asymptotic center for each subsequence $\left\{v_{n}\right\}$ of $\left\{x_{n}\right\}$. In fact, if $v \neq x$, then it follows Lemma 2.2 that $\lim _{n \rightarrow \infty} d\left(x_{n}, v\right)$ exists, and by the uniqueness of asymptotic centers, we have

$$
\limsup _{n \rightarrow \infty} d\left(v_{n}, v\right)<\limsup _{n \rightarrow \infty} d\left(v_{n}, x\right) \leqslant \limsup _{n \rightarrow \infty} d\left(x_{n}, x\right)<\limsup _{n \rightarrow \infty} d\left(x_{n}, v\right)=\limsup _{n \rightarrow \infty} d\left(v_{n}, v\right),
$$

which is a contradiction. Therefore $v=x$. Since $\left\{v_{n}\right\}$ is an arbitrary subsequence of $\left\{x_{n}\right\}, A_{k}\left(\left\{v_{n}\right\}\right)=\{x\}$ for all subsequence $\left\{v_{n}\right\}$ of $\left\{x_{n}\right\}$. Therefore, $\left\{x_{n}\right\} \Delta$-converges to a common fixed point $x$ of $\left\{T_{i}\right\}_{i \in \mathfrak{R}}$ and $\left\{S_{i}\right\}_{i \in \Re}$.

From Theorem 2.4, we have the following results.

Corollary 2.5. Let $\mathrm{K}$ be a nonempty closed and convex subset of a complete uniformly convex hyperbolic space $X$ with monotone modulus of uniform convexity $\eta$. For $i \in \mathfrak{R}$, let $\mathrm{T}_{\mathrm{i}}: \mathrm{K} \rightarrow \mathrm{K}$ be a uniformly $\mathrm{L}_{\mathrm{i}}$-Lipschitzian 
and $\left(\left\{\mu_{n}^{i}\right\},\left\{\xi_{n}^{i}\right\}, \rho^{i}\right)$-total quasi-asymptotically nonexpansive self-mapping, and $S_{i}: \mathrm{K} \rightarrow \mathrm{K}$ be a uniformly $\hat{\mathrm{L}}_{i^{-}}$ Lipschitzian and $\left(\left\{\hat{\mu}_{n}^{i}\right\},\left\{\hat{\xi}_{n}^{i}\right\}, \hat{\rho}^{i}\right)$-total asymptotically quasi-nonexpansive self-mapping, where constants $\mu_{n}^{i}, \xi_{n}^{i}$, $\rho^{i}, \hat{\mu}_{n}^{i}, \hat{\xi}_{n}^{i}, \hat{\rho}^{i}$ are the same as in Lemma 2.2. Assume that $\mathrm{F}=\bigcap_{i=1}^{r}\left(\mathrm{~F}\left(\mathrm{~T}_{i}\right) \cap \mathrm{F}\left(\mathrm{S}_{i}\right)\right) \neq \emptyset$ and conditions (i)-(ii) in Theorem 2.4 are satisfied, then the sequence $\left\{x_{n}\right\}$ defined in (1.2) $\Delta$-converges to a common fixed point $p \in F$.

Corollary 2.6. Let $\mathrm{K}$ be a nonempty closed and convex subset of a complete uniformly convex hyperbolic space $\mathrm{X}$ with monotone modulus of uniform convexity $\eta$. For $i \in \mathfrak{R}$, let $\mathrm{T}_{i}: \mathrm{K} \rightarrow \mathrm{K}$ be a uniformly $\mathrm{L}_{i}$-Lipschitzian and $\left\{\mathrm{k}_{n}^{i}\right\}-$ asymptotically nonexpansive mapping with $\sum_{n=1}^{\infty} \mathrm{k}_{n}^{i}<+\infty$, and $\mathrm{S}_{\mathrm{i}}: \mathrm{K} \rightarrow \mathrm{K}$ be a uniformly $\hat{\mathrm{L}}_{\mathrm{i}}$-Lipschitzian and $\left\{\hat{k}_{n}^{i}\right\}$-asymptotically nonexpansive mapping with $\sum_{n=1}^{\infty} \hat{k}_{n}^{i}<+\infty$. Assume that $\mathrm{F}=\bigcap_{i=1}^{r}\left(\mathrm{~F}\left(\mathrm{~T}_{i}\right) \cap \mathrm{F}\left(\mathrm{S}_{i}\right)\right) \neq \emptyset$. Then the sequence $\left\{x_{n}\right\}$ defined in (1.2) $\Delta$-converges to a common fixed point $p \in F$.

\section{Strong convergence}

In this section, we will prove strong convergence of the modified iterative approximating process (1.1) in hyperbolic spaces by using the related properties in Section 2.

Recall that a mapping $T: K \rightarrow X$ is semi-compact if every bounded sequence $\left\{x_{n}\right\} \subset K$, which satisfies $\mathrm{d}\left(\mathrm{x}_{\mathrm{n}}, \mathrm{T} \mathrm{x}_{\mathrm{n}}\right) \rightarrow 0$ as $\mathrm{n} \rightarrow \infty$, has a convergent subsequence.

Senter and Dotson [22] introduced condition (I) for $T: K \rightarrow X$ with $F(T) \neq \emptyset$ : if there exists a nondecreasing self-mapping on $[0,+\infty)$ with $f(0)=0$ and $f(t)>0$ for all $t \in(0,+\infty)$ such that $d(x, T x) \geqslant$ $f(d(x, F(T)))$ for all $x \in K$, where $d(x, F(T))=\inf \{d(x, y): y \in F(T)\}$. Further, for two finite families of self-maps, the corresponding conditions have been made recently in the literature $[4,6,17]$ as follows:

For $i \in \mathfrak{R}$, letting $\left\{\mathrm{T}_{i}\right\}_{\mathfrak{i} \in \mathfrak{R}}$ and $\left\{S_{i}\right\}_{i \in \mathfrak{R}}$ be two finite families of total asymptotically nonexpansive mappings on $\mathrm{K}$ with $\mathrm{F}=\bigcap_{i=1}^{n}\left(F\left(T_{i}\right) \cap F\left(S_{i}\right)\right) \neq \emptyset$, then the two families are said to satisfy:

(i) Condition (A) holds on $K$, if $d(x, T x) \geqslant f(d(x, F))$ or $d(x, S x) \geqslant f(d(x, F))$ for $x \in K$, holds for at least one $T \in\left\{T_{i}\right\}_{i \in \mathfrak{R}}$ or $S \in\left\{S_{i}\right\}_{i \in \mathfrak{R}}$, where $d(x, F)=\inf \{d(x, y): y \in F\}$;

(ii) Condition (B) holds on $K$, if $d\left(x, T_{i} x\right)+d\left(x, S_{i} x\right) \geqslant f(d(x, F))$ for $x \in K$ and $i \in \mathfrak{R}$.

Now we give the following conditions for two finite families of total asymptotically nonexpansive nonselfmappings $\left\{T_{i}\right\}_{i \in \mathfrak{R}}$ and $\left\{S_{i}\right\}_{i \in \mathfrak{R}}$ with $F=\bigcap_{i=1}^{n}\left(F\left(T_{i}\right) \cap F\left(S_{i}\right)\right) \neq \emptyset$ :

(iii) Condition $\left(C_{1}\right)$ holds on $K$ if, $\frac{1}{2 r}\left(\sum_{i=1}^{r} d\left(x, T_{i} x\right)+\sum_{i=1}^{r} d\left(x, S_{i} x\right)\right) \geqslant f(d(x, F))$ for $x \in K$;

(iv) Condition $\left(C_{2}\right)$ holds on $K$ if, $\frac{1}{2}\left(\max _{1 \leqslant i \leqslant r} d\left(x, T_{i} x\right)+\max _{1 \leqslant i \leqslant r} d\left(x, S_{i} x\right)\right) \geqslant f(d(x, F))$ for $x \in K$;

(v) Condition $\left(C_{3}\right)$ holds on $K$ if, $\max \left\{\max _{1 \leqslant i \leqslant r} d\left(x, T_{i} x\right), \max _{1 \leqslant i \leqslant r} d\left(x, S_{i} x\right)\right\} \geqslant f(d(x, F))$ for $x \in K$.

Remark 3.1. The conditions $(\mathbf{A}),(\mathbf{B})$ and $\left(C_{1}\right)-\left(C_{3}\right)$ are equivalent to the condition (I), if $T_{i}=S_{i}$ for $i \in \Re$. We shall use conditions $\left(C_{1}\right)-\left(C_{3}\right)$ to study strong convergence of the iteration (1.1).

In order to prove strong convergence of the iteration (1.1), we need the following lemma.

Lemma 3.2. Let $\mathrm{K}, \mathrm{X},\left\{\mathrm{T}_{i}\right\}_{\boldsymbol{i} \in \mathfrak{R}},\left\{\mathrm{S}_{\boldsymbol{i}}\right\}_{\boldsymbol{i} \in \mathfrak{R}}$, and $\left\{\mathrm{x}_{\mathrm{n}}\right\}$ be as in Theorem 2.4. Then $\left\{x_{n}\right\}$ converges strongly to some $\mathrm{p} \in \mathrm{F}$ if and only if

$$
\liminf _{n \rightarrow \infty} d\left(x_{n}, F\right)=0 .
$$

Proof. If $\left\{x_{n}\right\}$ converges strongly to $p \in F$, then $\lim _{n \rightarrow \infty} d\left(x_{n}, p\right)=0$. Since $0 \leqslant d\left(x_{n}, F\right) \leqslant d\left(x_{n}, p\right)$, we have $\liminf _{n \rightarrow \infty} d\left(x_{n}, F\right)=0$.

Conversely, suppose that $\liminf _{n \rightarrow \infty} d\left(x_{n}, F\right)=0$. It follows from Lemma 2.2 that $\lim _{n \rightarrow \infty} d\left(x_{n}, F\right)$ exists. Now $\liminf _{n \rightarrow \infty} d\left(x_{n}, F\right)=0$ reveals that $\lim _{n \rightarrow \infty} d\left(x_{n}, F\right)=0$.

Next, we show that $\left\{x_{n}\right\}$ is a Cauchy sequence. By last inequalities in the proof of Lemma 2.2

$$
d\left(x_{n+1}, p\right) \leqslant\left(1+M_{1} \mu_{n}\right) d\left(x_{n}, p\right)+M_{2} \xi_{n},
$$

and taking infimum on $p \in F$ on both sides in the above inequality, then we have

$$
d\left(x_{n+1}, F\right) \leqslant\left(1+M_{1} \mu_{n}\right) d\left(x_{n}, F\right)+M_{2} \xi_{n} .
$$


On account of $\sum_{n=1}^{\infty} \mu_{n}<\infty, \sum_{n=1}^{\infty} \xi_{n}<\infty$, set $e^{M_{1} \sum_{n=1}^{\infty} \mu_{n}}=M$. Since $\lim _{n \rightarrow \infty} d\left(x_{n}, F\right)=0$, for any given $\varepsilon>0$, there exists a positive integer $n_{0}$ such that

$$
\mathrm{d}\left(\mathrm{x}_{\mathrm{n}_{0}}, \mathrm{~F}\right)<\frac{\varepsilon}{4(M+1)} \text { and } \sum_{n=n_{0}}^{\infty} \xi_{\mathrm{n}}<\frac{\varepsilon}{2 M M_{2}} .
$$

The first inequality in (3.1) implies that there exists $p_{0} \in F$ such that $d\left(x_{n_{0}}, p_{0}\right)<\frac{\varepsilon}{2(M+1)}$. Hence, for any $n \geqslant n_{0}$ and $m \geqslant 1$, we have

$$
\begin{aligned}
d\left(x_{n_{0}+m}, x_{n_{0}}\right) \leqslant & d\left(x_{n_{0}+m}, p_{0}\right)+d\left(x_{n_{0}}, p_{0}\right) \\
\leqslant & {\left[e^{M_{1} \sum_{k=n_{0}}^{n_{0}+m-1} \mu_{k}}+1\right] d\left(x_{n_{0}}, p_{0}\right)+M_{2}\left[\xi_{n_{0}+m-1}\right.} \\
& \left.+\xi_{n_{0}+m-2} e^{M_{1} \mu_{n_{0}+m-1}}+\xi_{n_{0}+m-3} e^{M_{1} \sum_{k=n_{0}+m-2}^{n_{0}+m-1} \mu_{k}}+\cdots+\xi_{n_{0}} e^{M_{1} \sum_{k=n_{0}+1}^{n_{0}+m-1} \mu_{k}}\right] \\
\leqslant & (M+1) d\left(x_{n_{0}}, p_{0}\right)+M M_{2} \sum_{n=n_{0}}^{\infty} \xi_{n} \\
< & (M+1) \frac{\varepsilon}{2(M+1)}+M M_{2} \frac{\varepsilon}{2 M M_{2}}=\varepsilon
\end{aligned}
$$

This implies that $\left\{x_{n}\right\}$ is a Cauchy sequence in $X$. Sine $K$ is a closed subset of a complete hyperbolic space $X$, it is complete. We can assume that $\lim _{n \rightarrow \infty} x_{n}=q$, and $q \in K$. One can easily see that $F(T)$ is a close subset in $K$, so is $F(T)$. Since $\lim _{n \rightarrow \infty} d\left(x_{n}, F\right)=0$, we obtain $q \in F(T)$. This completes the proof.

We now establish strong convergence of the iteration schema (1.1) based on Lemma 3.2.

Theorem 3.3. Suppose that $\mathrm{K}, \mathrm{X},\left\{\mathrm{T}_{i}\right\}_{i \in \mathfrak{R}},\left\{S_{i}\right\}_{i \in \mathfrak{R}}$, and $\mathrm{F}$ be the same as in Theorem 2.4 , and $\left\{\mathrm{T}_{i}\right\}_{i \in \mathfrak{R}}$, and $\left\{S_{i}\right\}_{i \in \Re}$, satisfies condition $\left(\mathrm{C}_{1}\right.$ or $\mathrm{C}_{2}$ or $\left.\mathrm{C}_{3}\right)$. Then the sequence $\left\{\mathrm{x}_{\mathrm{n}}\right\}$ defined in (1.1) converges strongly to some $\mathrm{p} \in \mathrm{F}$.

Proof. It follows from Lemma 2.2 that $\lim _{n \rightarrow \infty} d\left(x_{n}, F\right)$ exists. Moreover, Lemma 2.3 implies that

$$
\lim _{n \rightarrow \infty} d\left(x_{n}, T_{i} x_{n}\right)=\lim _{n \rightarrow \infty} d\left(x_{n}, S_{i} x_{n}\right)=0
$$

for each $i \in \Re$. Thus, the condition $\left(C_{1}\right.$ or $C_{2}$ or $\left.C_{3}\right)$ guarantees that $\lim _{n \rightarrow \infty} f\left(d\left(x_{n}, F\right)\right)=0$. Since $f$ is nondecreasing with $f(0)=0$, it follows that $\lim _{n \rightarrow \infty} d\left(x_{n}, F\right)=0$. Thus, by Lemma 3.2, now we know that $\left\{x_{n}\right\}$ converges strongly to a common fixed point $p \in F$.

From Theorem 3.3, we have the following results.

Corollary 3.4. Let $\mathrm{K}, \mathrm{X},\left\{\mathrm{T}_{i}\right\}_{i \in \mathfrak{R}},\left\{\mathrm{S}_{i}\right\}_{i \in \mathfrak{R}}$, and $\mathrm{F}$ be the same as in Corollary 2.5. Suppose that $\left\{\mathrm{T}_{i}\right\}_{i \in \mathfrak{R}}$ and $\left\{\mathrm{S}_{i}\right\}_{i \in \mathfrak{R}}$ satisfy condition $\left(C_{1}\right.$ or $C_{2}$ or $\left.C_{3}\right)$. Then the sequence $\left\{x_{n}\right\}$ defined in (1.2) converges strongly to some $p \in F$.

Corollary 3.5. Assume that $\mathrm{K}, \mathrm{X},\left\{\mathrm{T}_{i}\right\}_{i \in \mathfrak{R}},\left\{\mathrm{S}_{\boldsymbol{i}}\right\}_{\mathfrak{i} \in \mathfrak{R}}$, and $\mathrm{F}$ are the same as in Corollary 2.6, and $\left\{\mathrm{T}_{i}\right\}_{i \in \mathfrak{R}}$ and $\left\{S_{i}\right\}_{i \in \Re}$ satisfy condition $\left(C_{1}\right.$ or $C_{2}$ or $\left.C_{3}\right)$. Then the sequence $\left\{x_{n}\right\}$ defined in (1.2) converges strongly to some $p \in F$.

Theorem 3.6. Let $\mathrm{K}, \mathrm{X},\left\{\mathrm{T}_{\mathrm{i}}\right\}_{\mathfrak{i} \in \mathfrak{R}},\left\{S_{i}\right\}_{\mathfrak{i} \in \mathfrak{R}}$, and $\mathrm{F}$ be the same as in Theorem 2.4. Suppose that either $\mathrm{T}_{\mathbf{l}} \in\left\{\mathrm{T}_{i}\right\}_{\mathrm{i} \in \mathfrak{R}}$ or $S_{l} \in\left\{S_{i}\right\}_{i \in \Re}$ is semi-compact. Then the sequence $\left\{x_{n}\right\}$ defined in (1.1) converges strongly to $p \in F$.

Proof. Let $T_{l} \in\left\{T_{i}\right\}_{i \in \mathfrak{R}}$ be semi-compact. By Lemma 2.3, we know that $\lim _{n \rightarrow \infty} d\left(T_{i} x_{n}, x_{n}\right)=0$ for all $i \in \mathfrak{R}$. By Lemma 2.2, $\left\{x_{n}\right\}$ is bounded and $T_{l}$ is semi-compact, there exists a subsequence $\left\{x_{n_{j}}\right\}$ of $\left\{x_{n}\right\}$ such that $x_{n_{j}} \rightarrow q$ as $j \rightarrow \infty$. By continuity of $T_{i}$ and Lemma 2.3, we obtain

$$
d\left(q, T_{i} q\right)=\lim _{j \rightarrow \infty} d\left(x_{n_{j}}, T_{i} x_{n_{j}}\right)=0, \quad i \in \mathfrak{R},
$$

which implies that $q$ is the common fixed point of $\left\{T_{i}\right\}_{i \in \mathfrak{R}}$. Similarly, we can show that $q$ is the common fixed point of $\left\{S_{i}\right\}_{i \in \mathfrak{R}}$. Hence, $q \in F$. Again, by Lemma 2.2, $\lim _{n \rightarrow \infty} d\left(x_{n}, q\right)$ exists. Therefore, $q$ is the strong limit of the sequence $\left\{x_{n}\right\}$. As a result, $\left\{x_{n}\right\}$ converges strongly to a point $q$. 
From Theorem 3.6, we have the following results.

Corollary 3.7. Let $\mathrm{K}, \mathrm{X},\left\{\mathrm{T}_{i}\right\}_{i \in \mathfrak{R}},\left\{\mathrm{S}_{i}\right\}_{i \in \mathfrak{R}}$, and $\mathrm{F}$ be the same as in Corollary 2.5. Suppose that either $\mathrm{T}_{\mathbf{l}} \in\left\{\mathrm{T}_{i}\right\}_{\mathrm{i} \in \mathfrak{R}}$ or $S_{l} \in\left\{S_{i}\right\}_{i \in \mathfrak{R}}$ is semi-compact. Then the sequence $\left\{x_{n}\right\}$ defined in (1.2) converges strongly to $\mathrm{p} \in \mathrm{F}$.

Corollary 3.8. Suppose that $\mathrm{K}, \mathrm{X},\left\{\mathrm{T}_{i}\right\}_{i \in \mathfrak{R}},\left\{\mathrm{S}_{i}\right\}_{i \in \mathfrak{R}}$, and $\left\{x_{n}\right\}$ be the same as in Corollary 2.6, and either $\mathrm{T}_{l} \in$ $\left\{T_{i}\right\}_{i \in \mathfrak{R}}$ or $S_{l} \in\left\{S_{i}\right\}_{i \in \mathfrak{R}}$ is semi-compact. Then the sequence $\left\{x_{n}\right\}$ defined in (1.2) converges strongly to $p \in F$.

Remark 3.9. The results presented in the paper extend and improve some recent results given in $[4,5,14$, $19,20,24,27,29,30,33]$. Some special cases are listed as follows.

(i) If $\left\{T_{i}\right\}_{i \in \mathfrak{R}}$ and $\left\{S_{i}\right\}_{i \in \Re}$ are asymptotically nonexpansive self-mappings on $K$, and the iterative process (1.1) reduce to (1.2), then Theorem 2.4, Lemma 3.2, and Theorems 3.3 and 3.6 reduce to Theorems 3.1 and 3.3-3.5 in [4], respectively.

(ii) when the uniformly convex hyperbolic spaces reduce to CAT(0) spaces, and the iterative process (1.1) is equivalent to (1.3), then Theorem 2.4, Lemma 3.2, and Theorem 3.3 become Theorems 3.1-3.3 proved by Thakur et al. [27], respectively.

(iii) Theorem 2.4, Lemma 3.2, and Theorems 3.3 and 3.6 are equivalent to Theorems 1-4 of [20], respectively, when $r=3$ and $\alpha_{i n}=0$ and $S_{1}=S_{2}=\cdots=S_{r}=T$ are self-mappings.

(iv) If the uniformly convex hyperbolic spaces reduce to CAT(0) spaces, $r=3, \alpha_{i n}=0$, and $S_{1}^{n}=S_{2}^{n}=$ $\cdots=\mathrm{S}_{\mathrm{r}}^{n}=\mathrm{T}$, where $\mathrm{T}$ is a nonexpansive mappings on $\mathrm{K} \subset \mathrm{X}$, then Theorem 2.4, Lemma 3.2, and Theorem 3.3 reduce to Theorems 1-3 in [19], respectively.

\section{Acknowledgment}

This work was partially supported by Sichuan Province Cultivation Fund Project of Academic and Technical Leaders, the Scientific Research Project of Sichuan University of Science and Engineering (2015RC07) and the Scientific Research Fund of Sichuan Provincial Education Department (16ZA0256).

\section{References}

[1] R. P. Agarwal, D. O'Regan, D. R. Sahu, Iterative construction of fixed points of nearly asymptotically nonexpansive mappings, J. Nonlinear convex Anal., 8 (2007), 61-79. 1.1

[2] B. Ali, Convergence theorems for finite families of total asymptotically nonexpansive mappings in hyperbolic spaces, Fixed Point Theory Appl., 2016 (2016), 13 pages. 1.1

[3] M. R. Bridson, A. Haefliger, Metric spaces of non-positive curvature, Grundlehren der Mathematischen Wissenschaften [Fundamental Principles of Mathematical Sciences], Springer-Verlag, Berlin, (1999). 1, 1.6

[4] H. Fukhar-ud-din, M. A. A. Khan, Convergence analysis of a general iteration schema of nonlinear mappings in hyperbolic spaces, Fixed Point Theory Appl., 2013 (2013), 18 pages. 1.1, 1.2, 3, 3.9, i

[5] S.H. Khan, A Picard-Mann hybrid iterative process, Fixed Point Theory Appl., 2013 (2013), 10 pages. 1.1, 1.4, 3.9

[6] S. H. Khan, H. Fukhar-ud-din, Weak and strong convergence of a scheme with errors for two nonexpansive mappings, Nonlinear Anal., 61 (2005), 1295-1301. 3

[7] A. R. Khan, H. Fukhar-ud-din, M. A. A. Khan, An implicit algorithm for two finite families of nonexpansive maps in hyperbolic spaces, Fixed Point Theory Appl., 2012 (2012), 12 pages. 1.11

[8] A. R. Khan, M. A. Khamsi, H. Fukhar-ud-din, Strong convergence of a general iteration scheme in CAT(0) spaces, Nonlinear Anal., 74 (2011), 783-791. 1.1

[9] U. Kohlenbach, Some logical metatheorems with applications in functional analysis, Trans. Amer. Math. Soc., 357 (2005), 89-128. 1, 1

[10] W. A. Kirk, B. Panyanak, A concept of convergence in geodesic spaces, Nonlinear Anal., 68 (2008), 3689-3696. 2

[11] P. K. F. Kuhfittig, Common fixed points of nonexpansive mappings by iteration, Pacific J. Math., 97 (1981), 137-139. 1

[12] P. Kumam, G. S. Saluja, H. K. Nashine, Convergence of modified S-iteration process for two asymptotically nonexpansive mappings in the intermediate sense in CAT(0) spaces, J. Inequal. Appl., 2014 (2014), 15 pages. 1.1

[13] L. Leuştean, Nonexpansive iterations in uniformly convex W-hyperbolic spaces, Nonlinear analysis and optimization I, Nonlinear analysis, Contemp. Math., Israel Math. Conf. Proc., Amer. Math. Soc., Providence, RI, 513 (2010), 193-209. 1.7, 1, 1.10

[14] Y. Li, L. H. Bo, $\Delta$-convergence analysis of improved Kuhfittig iterative for asymptotically nonexpansive nonself-mappings in $\mathrm{W}$-hyperbolic spaces, J. Inequal. Appl., 2014 (2014), 9 pages. 1.1, 3.9 
[15] T. C. Lim, Remarks on some fixed point theorems, Proc. Amer. Math. Soc., 60 (1976), 179-182. 2

[16] M.A. Noor, New approximation schemes for general variational inequalities, J. Math. Anal. Appl., 251 (2000), $217-229$. $1.1,1.3$

[17] S. Plubtieng, K. Ungchittrakool, R. Wangkeeree, Implicit iterations of two finite families for nonexpansive mappings in Banach spaces, Numer. Funct. Anal. Optim., 28 (2007), 737-749. 3

[18] S. Reich, I. Shafrir, Nonexpansive iterations in hyperbolic spaces, Nonlinear Anal., 15 (1990), 537-558. 1

[19] A. Şahin, M. Başarır, On the strong and $\Delta$-convergence of SP-iteration on CAT(0) space, J. Inequal. Appl., 2013 (2013), 10 pages. $1.1,1.3,3.9$, iv

[20] A. Şahin, M. Başarır, Some convergence results for modified SP-iteration scheme in hyperbolic spaces, Fixed Point Theory Appl., 2014 (2014), 11 pages. 1.3, 3.9, iii

[21] J. Schu, Weak and strong convergence to fixed points of asymptotically nonexpansive mappings, Bull. Austral. Math. Soc., 43 (1991), 153-159. 1.1, 1.5

[22] H. F. Senter, W. G. Dotson Jr., Approximating fixed points of nonexpansive mappings, Proc. Amer. Math. Soc., 44 (1974), 375-380. 3

[23] T. Shimizu, W. Takahashi, Fixed points of multivalued mappings in certain convex metric spaces, Topol. Methods Nonlinear Anal., 8 (1996), 197-203. 1.6

[24] S. Suantai, Weak and strong convergence criteria of Noor iterations for asymptotically nonexpansive mappings, J. Math. Anal. Appl., 311 (2005), 506-517. 1.1, 1.3, 3.9

[25] W. Takahashi, A convexity in metric space and nonexpansive mappings, I. Kōdai Math. Sem. Rep., 22 (1970), 142-149. 1

[26] K.-K. Tan, H. K. Xu, Approximating fixed points of nonexpansive mappings by the Ishikawa iteration process, J. Math. Anal. Appl., 178 (1993), 301-308. 1.12

[27] B. S. Thakur, D. Thakur, M. Postolache, Modified Picard-Mann hybrid iteration process for total asymptotically nonexpansive mappings, Fixed Point Theory Appl., 2015 (2015), 11 pages. 1.1, 1.4, 3.9, ii

[28] L.-L. Wan, $\Delta$-convergence for mixed-type total asymptotically nonexpansive mappings in hyperbolic spaces, J. Inequal. Appl., 2013 (2013), 8 pages.

[29] L.-L. Wan, Demiclosed principle and convergence theorems for total asymptotically nonexpansive nonself mappings in hyperbolic spaces, Fixed Point Theory Appl., 2015 (2015), 10 pages. 1.9, 1.13, 2, 3.9

[30] L. Wang, S.-S. Chang, Z.-L. Ma, Convergence theorems for total asymptotically nonexpansive non-self mappings in CAT $(0)$ spaces, J. Inequal. Appl., 2013 (2013), 10 pages. 1.9, 3.9

[31] T.-J. Xiong, H.-Y. Lan, Convergence analysis of new iterative approximating schemes with errors for total asymptotically nonexpansive mappings in hyperbolic spaces, J. Comput. Anal. Appl., 20 (2016), 902-913. 1.1, 1

[32] L. Yang, F. H. Zhao, Strong and $\Delta$-convergence theorems for total asymptotically nonexpansive nonself mappings in CAT(0) spaces, J. Inequal. Appl., 2013 (2013), 17 pages. 1.9, 2

[33] I. Yildirim, M. Özdemir, Approximating common fixed points of asymptotically quasi-nonexpansive mappings by a new iterative process, Arab. J. Sci. Eng., 36 (2011), 393-403. 1.1, 3.9 